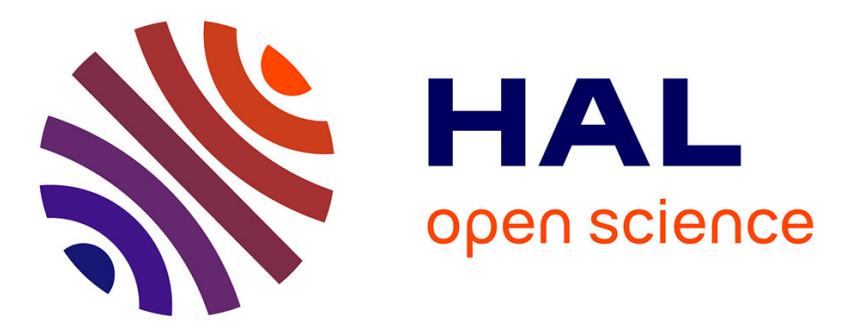

\title{
A new assumed strain solid-shell formulation "SHB6" for the six-node prismatic finite element
} Vuong-Dieu Trinh, Farid Abed-Meraim, Alain Combescure

\section{To cite this version:}

Vuong-Dieu Trinh, Farid Abed-Meraim, Alain Combescure. A new assumed strain solid-shell formulation "SHB6" for the six-node prismatic finite element. Journal of Mechanical Science and Technology, 2011, 25 (9), pp.2345-2364. 10.1007/s12206-011-0710-7 . hal-01206008

\section{HAL Id: hal-01206008 \\ https://hal.science/hal-01206008}

Submitted on 28 Sep 2015

HAL is a multi-disciplinary open access archive for the deposit and dissemination of scientific research documents, whether they are published or not. The documents may come from teaching and research institutions in France or abroad, or from public or private research centers.
L'archive ouverte pluridisciplinaire HAL, est destinée au dépôt et à la diffusion de documents scientifiques de niveau recherche, publiés ou non, émanant des établissements d'enseignement et de recherche français ou étrangers, des laboratoires publics ou privés. 


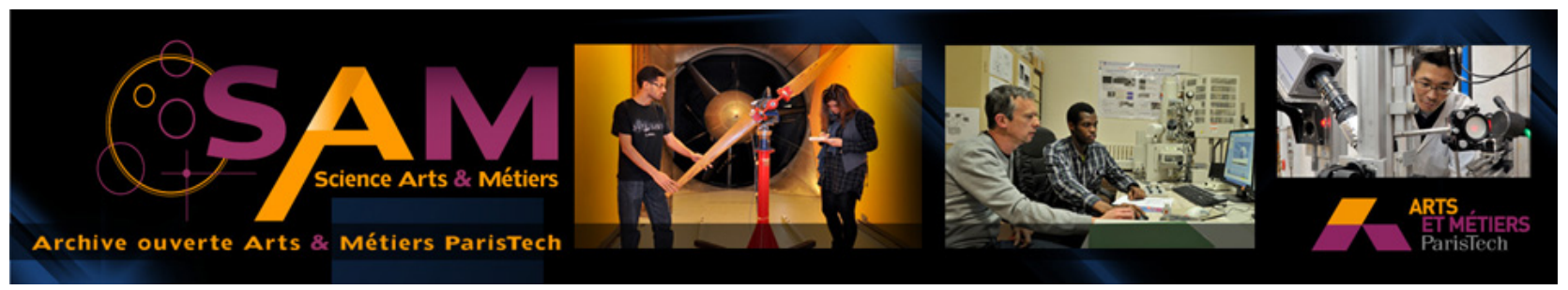

\section{Science Arts \& Métiers (SAM)}

is an open access repository that collects the work of Arts et Métiers ParisTech researchers and makes it freely available over the web where possible.

This is an author-deposited version published in: http://sam.ensam.eu

Handle ID: .http://hdl.handle.net/10985/10193

\section{To cite this version :}

Vuong-Dieu TRINH, Farid ABED-MERAIM, Alain COMBESCURE - A new assumed strain solidshell formulation "SHB6" for the six-node prismatic finite element - Journal of Mechanical Science and Technology - Vol. 25, n9, p.2345-2364 - 2011 


\title{
A new assumed strain solid-shell formulation "SHB6" for the six-node prismatic finite element ${ }^{\dagger}$
}

\author{
Vuong-Dieu Trinh ${ }^{1,2}$, Farid Abed-Meraim, ${ }^{1, *}$ and Alain Combescure ${ }^{3}$ \\ ${ }^{1}$ LEM3, UMR CNRS 7239, Arts et Métiers ParisTech, 4 rue A. Fresnel, 57078 Metz Cedex 03, France \\ ${ }^{2}$ LaMSID, UMR EDF/CNRS 2832, EDF R\&D, 1 avenue du Général de Gaulle, 92141 Clamart Cedex, France \\ ${ }^{3}$ LaMCoS, UMR CNRS 5259, INSA de Lyon, 18-20 rue des Sciences, 69621 Villeurbanne Cedex, France
}

\begin{abstract}
This paper presents the development of a new prismatic solid-shell finite element, denoted SHB6, obtained using a purely threedimensional approach. This element has six nodes with displacements as the only degrees of freedom, and only requires two integration points distributed along a preferential direction, designated as the "thickness". Although geometrically three-dimensional, this element can be conveniently used to model thin structures while taking into account the various phenomena occurring across the thickness. A reduced integration scheme and specific projections of the strains are introduced, based on the assumed-strain method, in order to improve performance and to eliminate most locking effects. It is first shown that the adopted in-plane reduced integration does not generate "hourglass" modes, but the resulting SHB6 element exhibits some shear and thickness-type locking. This is common in linear triangular elements, in which the strain is constant. The paper details the formulation of this element and illustrates its capabilities through a set of various benchmark problems commonly used in the literature. In particular, it is shown that this new element plays a useful role as a complement to the SHB8PS hexahedral element, which enables one to mesh arbitrary geometries. Examples using both SHB6 and SHB8PS elements demonstrate the advantage of mixing these two solid-shell elements.
\end{abstract}

Keywords: Assumed-strain solid-shell SHB6; Benchmark problems; Buckling; Hourglass; Shear and thickness locking; Six-node prism

\section{Introduction}

Accuracy and efficiency are the main attributes expected in computer simulations based on the finite element method. Indeed, the ever-growing reliance on commercial software packages to solve actual engineering problems has become widespread and is a universally acknowledged necessity in many sectors of the industry. In practical engineering situations, shell structures are found in a wide range of applications, making the accurate analysis of complex shell structures a matter of primary concern to engineers and designers. This issue is even more crucial when structures, in which thin structural zones and thicker three-dimensional parts simultaneously coexist, need to be discretized for finite element (FE) analyses. For more than three decades, considerable progress has been made in the development of fast and reliable shell elements for such structural applications. Numerous efficient plate and shell elements have been developed based on mixed

\footnotetext{
Maenghyo Cho

*Corresponding author. Tel.: +33 3873754 79, Fax.: +33 387375470

E-mail address: farid.abed-meraim@ensam.eu
}

formulations or shear projection techniques in order to avoid locking problems. For this purpose, two approaches have been mainly used to formulate shell theories, which range from the degenerated three-dimensional concept originated by Ahmad [1], and subsequently adopted by Hughes and Liu [2], to the more classical shell descriptions (see, e.g., Bathe and Dvorkin [3, 4], Simo et al. [5], Cheung and Chen [6], Onate and Castro [7], Wriggers and Gruttmann [8], Ayad et al. [9], Chapelle and Bathe [10], Zeng and Combescure [11]). However, the planestress assumptions, which are the most commonly adopted in these formulations, require special treatment for the integration of the constitutive equations and represent one of the major drawbacks of shell derivations. When these assumed simplifications are not acceptable, or if advanced constitutive behavior models need to be implemented in a fully threedimensional manner, more sophisticated shell theories that take thickness changes into account have to be developed. Shell formulations of this kind, accounting for through-thethickness stretching have been proposed by Parish [12], Buechter et al. [13], Betsch et al. [14], Bischoff and Ramm [15], Brank et al. [16], and Cardoso and Yoon [17]. Another difficulty with shell elements, besides their more complex and 
elaborate formulation, is the need for a special treatment of the rotational degrees of freedom when dealing with large rotations. In addition, connecting shell elements with threedimensional solid elements is another intricacy that requires kinematic assumptions at the interface resulting in excessively stiff behavior.

For the above-mentioned reasons, and because most reallife structures contain both three-dimensional and structural zones, continuum-based elements are desirable for a wide range of applications. Elements that behave well in both continuum and structural problems would considerably simplify the modeling of such structures and avoid arbitrary definitions of separation zones (e.g., continuum/structural) as well as the intricacies of connecting different types of elements (e.g., shell/continuum). Furthermore, continuum-based elements have many other advantages: the avoidance of complex shelltype kinematics, the use of general three-dimensional constitutive models, direct calculation of thickness (strain) variations, easy treatment of large rotations along with simple updating of configurations, straightforward connections with threedimensional elements because displacements are the only degrees of freedom, and natural contact conditions on both sides of the structure. However, classical low-order solid elements are known to exhibit locking effects in thin structure applications and bending-dominated problems. Due to efficiency requirements, as well as compatibility with contact algorithms, remedies to these locking effects should be found while maintaining low-order interpolation procedures, and preferably with reduced integration rules. In this regard, considerable attention has been focused on the idea of modifying three-dimensional elements so that they are able to accurately reproduce the behavior of thin shell structures. The pioneering authors dealing with thin structure modeling by means of three-dimensional elements without rotational degrees of freedom include Graf et al. [18], Xu and Cai [19], Sze and Ghali [20], Kim and Lee [21], and Buragohain and Ravichandran [22].

More recently, the solid-shell finite element concept has emerged as an interesting way to combine the advantages of both solid and shell elements. Since they are intended to compete with shell elements, solid-shell elements are expected to have two key features: they contain only displacements as degrees of freedom, and they are able to reproduce the behavior of thin structures by means of a single layer of elements through the thickness. In the last decade, several examples of such formulations have been proposed, and can be found in Domissy [23], Cho et al. [24], Hauptmann and Schweizerhof [25], Lemosse [26], Sze and Yao [27], Hauptmann et al. [28], Sze and Chan [29], Abed-Meraim and Combescure [30, 31], Legay and Combescure [32], Vu-Quoc and Tan [33], Sze et al. [34], Chen and $\mathrm{Wu}$ [35], Kim et al. [36], Alves de Sousa et al. [37-39], and Reese [40]. It should be noted that most of the methods developed earlier were based on the enhanced assumed strain method proposed by Simo and co-workers (Simo and Rifai [41], Simo and Armero [42], Simo et al. [43]), and consisted of either the use of a conventional integration scheme with appropriate control of all locking phenomena or the application of a reduced integration technique with associated hourglass control. Both approaches have been extensively investigated and evaluated in various structural applications, as reported in the works of Dvorkin and Bathe [44], Belytschko and Bindeman [45], Zhu and Cescotto [46], Wriggers and Reese [47], Klinkel and Wagner [48], Klinkel et al. [49], Wall et al. [50], Reese et al. [51], and Puso [52].

On the other hand, this successful development of hexahedral solid-shell elements has made the existence of prismatic solid-shell elements desirable in order to mesh arbitrary structures easily and automatically. Indeed, to be able to mesh arbitrarily-shaped geometries, and with the advent of free mesh generation tools that do not only generate hexahedrons, the development of triangular-based solid-shell three-dimensional elements is necessary. This represents the main motivation of the present work, in which a new prismatic solid-shell element, denoted SHB6, is developed. This element is formulated following a scheme similar to that used for the recently developed hexahedral solid-shell element, denoted SHB8PS, and based on a purely three-dimensional approach [31, 32]. Recall that in order to improve the efficiency of this element and to alleviate some shear and thickness locking phenomena, the reduced integration technique was used. This method consists of using fewer integration points than required for a complete integration scheme in calculating the element stiffness matrix. This reduced integration technique was described, for example, in Zienkiewicz et al. [53], Pawsey and Clough [54], Hughes et al. [55], and Flanagan and Belytschko [56], among many others. However, this method is known to produce unstable elements characterized by the presence of zero-energy modes, also known as "hourglass" patterns. An effective and physical stabilization technique, following the approach of Belytschko and Bindeman [45], was used for the SHB8PS element in order to eliminate these spurious zero-energy modes.

In the present paper, the detailed formulation of the SHB6 solid-shell element is given. This is an isoparametric six-node prismatic element with linear interpolation using an in-plane reduced integration scheme. Similar to the SHB8PS, the nodal displacements are the only degrees of freedom, and it is provided with a set of integration points distributed in a preferential direction, designated as the "thickness". It is worth noting that the proposed formulation is valid for any set of integration points located along the thickness direction and comprising at least two integration points. The analysis of the stiffness matrix rank deficiency revealed that, in contrast to the SHB8PS element, the SHB6 element has no hourglass modes; therefore, stabilization is not required. Nevertheless, we propose modifications, based on the well-known assumed-strain method [45], for the discrete gradient operator of the element in order to improve its convergence rate. The remainder of this paper details the formulation of this element and illustrates its capabilities through a set of benchmark problems commonly used 


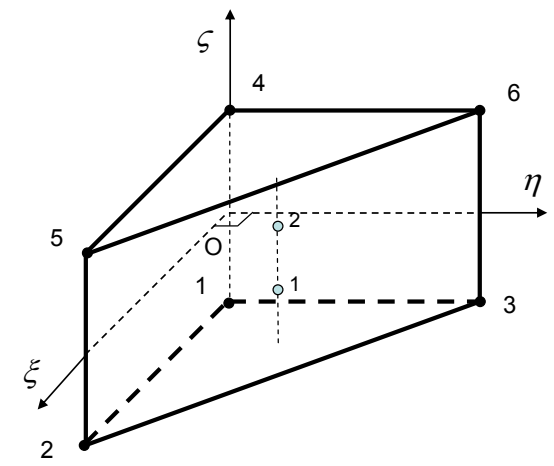

Fig. 1. Reference geometry of the element and example of integration points.

in the literature. In particular, it is shown that this new element plays a useful role as a complement to the SHB8PS hexahedral element, which enables one to mesh arbitrary geometries. Examples using both SHB6 and SHB8PS elements demonstrate the advantage of mixing these two solid-shell elements.

\section{Formulation of the prismatic continuum shell ele- ment SHB6}

The SHB6 element is a purely three-dimensional six-node prism with three displacement degrees of freedom at each node, and a preferential direction, called the thickness, normal to the mean plane of the triangle. The numerical integration across the thickness is based on a set of $n_{\text {int }}$ Gauss points, whereas the integration in the two other directions is carried out with a single Gauss point located at the centroid of the element. Note that two integration points are sufficient for both providing a rank sufficient element and dealing with elastic problems, as will be shown in Section 3. Fig. 1 shows the reference geometry of the SHB6 element as well as the location of its integration points in the case of two-point quadrature.

The element is formulated in the local axes of the mean plane. The $x, y, z$ (or $1,2,3$ ) directions are parallel to the $\xi$, $\eta, \zeta$ axes, respectively.

\subsection{Kinematics and finite element interpolation}

In the prismatic element, the spatial coordinates $x_{i}$ of point $\mathbf{x}$ are related to the nodal coordinates $x_{i J}$ of the nodes $J$, through the shape functions $N_{J}$, by:

$$
x_{i}(\xi, \eta, \zeta)=x_{i J} N_{J}(\xi, \eta, \zeta)=\sum_{J=1}^{6} x_{i J} N_{J}(\xi, \eta, \zeta)
$$

From now on, except when mentioned otherwise, we will follow the summation convention for repeated indices. Lowercase indices $i$ vary between one and three and represent the directions of the spatial coordinates. Uppercase indices $J \quad$ vary between one and six and correspond to the nodes of the element.
The same shape functions are used to define the $i^{\text {th }}$ component of the displacement field of the element $\left(u_{i}\right)$ as a function of the nodal displacements $u_{i J}$ :

$$
u_{i}(\xi, \eta, \zeta)=u_{i J} N_{J}(\xi, \eta, \zeta)
$$

\subsection{Strain-displacement relation and discrete gradient op- erator}

The interpolation of the displacement field, Eq. (2), enables one to express the strain field as a function of the nodal displacements. The linear part of the strain tensor becomes:

$$
\varepsilon_{i k}=\frac{1}{2}\left(u_{i, k}+u_{k, i}\right)=\frac{1}{2}\left(u_{i J} N_{J, k}+u_{k J} N_{J, i}\right) .
$$

In the case of the six-node prismatic element, the isoparametric shape functions are known explicitly:

$$
\left\{\begin{array}{l}
N_{1}(\xi, \eta, \zeta)=\frac{1}{2}(1-\zeta)(1-\xi-\eta) \\
N_{2}(\xi, \eta, \zeta)=\frac{1}{2}(1-\zeta) \xi \\
N_{3}(\xi, \eta, \zeta)=\frac{1}{2}(1-\zeta) \eta \\
N_{4}(\xi, \eta, \zeta)=\frac{1}{2}(1+\zeta)(1-\xi-\eta) \\
N_{5}(\xi, \eta, \zeta)=\frac{1}{2}(1+\zeta) \xi \\
N_{6}(\xi, \eta, \zeta)=\frac{1}{2}(1+\zeta) \eta \\
\xi \in[0,1], \eta \in[0,1-\xi], \zeta \in[-1,1] .
\end{array}\right.
$$

Let $\mathbf{N}$ denote the vector of the six shape functions. These shape functions transform a right regular prism in the reference space $(\xi, \eta, \zeta)$ into an arbitrary prism in the $\left(x_{1}, x_{2}, x_{3}\right)$ space. By combining Eqs. (1), (2), and (4), one can expand the strain field into a constant term, linear terms in $x_{i}$, and additional terms involving some functions $h_{\alpha}$. We end up with the following expression:

$$
\left\{\begin{array}{l}
u_{i}(\xi, \eta, \zeta, x, y, z)=a_{0 i}+a_{1 i} x+a_{2 i} y+a_{3 i} z+c_{1 i} h_{1}+c_{2 i} h_{2} \\
i=1,2,3 \\
h_{1}=\zeta \eta, h_{2}=\zeta \xi .
\end{array}\right.
$$

The coefficients $a_{k i}$ and $c_{\alpha i}$ are constants that depend on the geometry of the element and will be determined subsequently. By evaluating Eq. (5) at the element nodes, one obtains the three displacements $i$ at each of the six nodes of the element through:

$$
\mathbf{d}_{i}=a_{0 i} \mathbf{s}+a_{1 i} \mathbf{x}_{1}+a_{2 i} \mathbf{x}_{2}+a_{3 i} \mathbf{x}_{3}+c_{1 i} \mathbf{h}_{1}+c_{2 i} \mathbf{h}_{2} \quad ; \quad i=1,2,3 .
$$

In Eq. (6), $\mathbf{d}_{i}$ and $\mathbf{x}_{i}$ are six-component vectors which contain, respectively, the three displacements and the three coordinates of the six nodes of the element, and are defined by: 


$$
\left\{\begin{array}{l}
\mathbf{d}_{i}^{T}=\left(u_{i 1}, u_{i 2}, u_{i 3}, u_{i 4}, u_{i 5}, u_{i 6}\right) \\
\mathbf{x}_{i}^{T}=\left(x_{i 1}, x_{i 2}, x_{i 3}, x_{i 4}, x_{i 5}, x_{i 6}\right)
\end{array}\right.
$$

Vectors $\mathbf{s}$ and $\mathbf{h}_{\alpha}(\alpha=1,2)$ are given by:

$$
\left\{\begin{array}{l}
\mathbf{s}^{T}=(1,1,1,1,1,1) \\
\mathbf{h}_{1}^{T}=(0,0,-1,0,0,1) \\
\mathbf{h}_{2}^{T}=(0,-1,0,0,1,0) .
\end{array}\right.
$$

Let $\mathbf{b}_{i}(i=1,2,3)$ be the Hallquist vectors [57] given by:

$$
\mathbf{b}_{i}=\mathbf{N}_{, i}(\mathbf{0})=\frac{\partial \mathbf{N}}{\partial x_{i \mid \xi=\eta=\zeta=0}} \quad i=1,2,3 \quad \text { Hallquist Form }
$$

The explicit expressions of the derivatives of the shape functions evaluated at the origin of the $(\xi, \eta, \zeta)$ frame are derived in Appendix A. This explicit derivation allows us to demonstrate the following orthogonality properties, which involve vectors $\mathbf{b}_{i}$ and vectors $\mathbf{x}_{i}^{T}=\left(x_{i 1}, x_{i 2}, \ldots, x_{i 6}\right)$, $\mathbf{d}_{i}^{T}=\left(u_{i 1}, u_{i 2}, \ldots, u_{i 6}\right), \mathbf{s}, \mathbf{h}_{1}, \mathbf{h}_{2}$ :

$$
\begin{cases}\mathbf{b}_{i}^{T} \cdot \mathbf{h}_{\alpha}=0, & \mathbf{b}_{i}^{T} \cdot \mathbf{s}=0 \quad, \quad \mathbf{b}_{i}^{T} \cdot \mathbf{x}_{j}=\delta_{i j} \\ \mathbf{h}_{\alpha}^{T} \cdot \mathbf{s}=0, & \mathbf{h}_{\alpha}^{T} \cdot \mathbf{h}_{\beta}=2 \delta_{\alpha \beta} \\ i, j=1, \ldots, 3 & \alpha, \beta=1,2 .\end{cases}
$$

These orthogonality relations enable one to determine constants $a_{k i}$ and $c_{\alpha i}$ through the scalar product of Eq. (6) by $\mathbf{b}_{k}^{T}$ and $\mathbf{h}_{\alpha}^{T}$, respectively. Finally, one obtains:

$$
\left\{\begin{array}{l}
a_{k i}=\mathbf{b}_{k}^{T} \cdot \mathbf{d}_{i} \quad, \quad c_{\alpha i}=\boldsymbol{\gamma}_{\alpha}^{T} \cdot \mathbf{d}_{i} \\
\text { where: } \quad \gamma_{\alpha}=\frac{1}{2}\left[\mathbf{h}_{\alpha}-\sum_{j=1}^{3}\left(\mathbf{h}_{\alpha}^{T} \cdot \mathbf{x}_{j}\right) \mathbf{b}_{j}\right] .
\end{array}\right.
$$

In the end, the displacement field takes the very convenient form:

$$
u_{i}=a_{0 i}+\left(x_{1} \mathbf{b}_{1}^{T}+x_{2} \mathbf{b}_{2}^{T}+x_{3} \mathbf{b}_{3}^{T}+h_{1} \gamma_{1}^{T}+h_{2} \gamma_{2}^{T}\right) \cdot \mathbf{d}_{i}
$$

Differentiating this last equation with respect to $x_{j}$ yields the displacement gradient as follows:

$$
u_{i, j}=\left(\mathbf{b}_{j}^{T}+\sum_{\alpha=1}^{2} h_{\alpha, j} \gamma_{\alpha}^{T}\right) \cdot \mathbf{d}_{i}=\left(\mathbf{b}_{j}^{T}+h_{\alpha, j} \gamma_{\alpha}^{T}\right) \cdot \mathbf{d}_{i}
$$

This allows one to express the discrete gradient operator relating the strain field to the nodal displacements as:

$$
\begin{gathered}
\nabla_{s}(\mathbf{u})=\mathbf{B} \cdot \mathbf{d} \\
\text { where: } \nabla_{s}(\mathbf{u})=\left[\begin{array}{c}
u_{x, x} \\
u_{y, y} \\
u_{z, z} \\
u_{x, y}+u_{y, x} \\
u_{y, z}+u_{z, y} \\
u_{x, z}+u_{z, x}
\end{array}\right], \quad \mathbf{d}=\left[\begin{array}{c}
\mathbf{d}_{x} \\
\mathbf{d}_{y} \\
\mathbf{d}_{z}
\end{array}\right] .
\end{gathered}
$$

This discrete gradient operator finally takes the following practical matrix form:

$$
\mathbf{B}=\left[\begin{array}{ccc}
\mathbf{b}_{x}^{T}+h_{\alpha, x} \gamma_{\alpha}^{T} & \mathbf{0} & \mathbf{0} \\
\mathbf{0} & \mathbf{b}_{y}^{T}+h_{\alpha, y} \gamma_{\alpha}^{T} & \mathbf{0} \\
\mathbf{0} & \mathbf{0} & \mathbf{b}_{z}^{T}+h_{\alpha, z} \gamma_{\alpha}^{T} \\
\mathbf{b}_{y}^{T}+h_{\alpha, y} \gamma_{\alpha}^{T} & \mathbf{b}_{x}^{T}+h_{\alpha, x} \gamma_{\alpha}^{T} & \mathbf{0} \\
\mathbf{0} & \mathbf{b}_{z}^{T}+h_{\alpha, z} \gamma_{\alpha}^{T} & \mathbf{b}_{y}^{T}+h_{\alpha, y} \gamma_{\alpha}^{T} \\
\mathbf{b}_{z}^{T}+h_{\alpha, z} \gamma_{\alpha}^{T} & \mathbf{0} & \mathbf{b}_{x}^{T}+h_{\alpha, x} \gamma_{\alpha}^{T}
\end{array}\right] .
$$

One should note that the above expression of the discrete gradient operator is very useful, since it allows each of the non-constant strain modes to be handled separately so that an assumed-strain field can be easily and conveniently built. Moreover, it is easy to show that the $\gamma_{\alpha}$ vectors that enter the expression of matrix $\mathbf{B}$ verify the following orthogonality conditions:

$$
\boldsymbol{\gamma}_{\alpha}^{T} \cdot \mathbf{x}_{j}=0, \quad \boldsymbol{\gamma}_{\alpha}^{T} \cdot \mathbf{h}_{\beta}=\delta_{\alpha \beta} .
$$

These properties will be helpful in the subsequent hourglass stability analysis of the SHB6 element. They will also help in choosing an appropriate assumed-strain field.

\subsection{Hu-Washizu principle and variational formulation}

The extension of the weak form of the $\mathrm{Hu}$-Washizu variational principle to nonlinear solid mechanics was introduced by Fish and Belytschko [58]. For a single finite element, this three-field variational principle reads:

$$
\delta \pi(\mathbf{v}, \dot{\overline{\boldsymbol{\varepsilon}}}, \overline{\boldsymbol{\sigma}})=\int_{\Omega_{e}} \delta \dot{\overline{\boldsymbol{\varepsilon}}}^{T} \cdot \boldsymbol{\sigma} d \Omega+\delta \int_{\Omega_{e}} \overline{\boldsymbol{\sigma}}^{T} \cdot\left(\nabla_{s}(\mathbf{v})-\dot{\overline{\boldsymbol{\varepsilon}}}\right) d \Omega-\delta \dot{\mathbf{d}}^{T} \cdot \mathbf{f}^{e x t}=0 .
$$

In Eq. (17), $\delta$ denotes a variation, $\mathbf{v}$ the velocity field, $\dot{\bar{\varepsilon}}$ the assumed-strain rate, $\overline{\boldsymbol{\sigma}}$ the interpolated stress, $\boldsymbol{\sigma}$ the stress deduced from the constitutive law, $\dot{d}$ the nodal velocities, $\mathbf{f}^{\text {ext }}$ the external nodal forces, and $\nabla_{s}(\mathbf{v})$ the symmet- 
ric part of the gradient of the velocity field. Note that the superposed dot in the assumed-strain rate symbol, $\dot{\bar{\varepsilon}}$, does not indicate a time derivative.

The assumed-strain formulation used in the following construction of the element is based on a simplified form of the $\mathrm{Hu}-$ Washizu variational principle as described by Simo and Hughes [59]. In this simplified form, the interpolated stress is chosen to be orthogonal to the difference between the symmetric part of the velocity gradient and the assumed-strain rate. As a result, the second term in the right-hand side of Eq. (17) vanishes, and one obtains:

$$
\delta \pi(\dot{\overline{\boldsymbol{\varepsilon}}})=\int_{\Omega_{e}} \delta \dot{\overline{\boldsymbol{\varepsilon}}}^{T} \cdot \boldsymbol{\sigma} d \Omega-\delta \dot{\mathbf{d}}^{T} \cdot \mathbf{f}^{e x t}=0
$$

In this form, the variational principle is independent of the stress interpolation, since the interpolated stress is no longer present; therefore, it does not need to be defined. Hence, the discrete equations only require the interpolation of the velocity and of the assumed-strain field over the element. The assumed-strain rate $\dot{\bar{\varepsilon}}$ is expressed in terms of a $\overline{\mathbf{B}}$ matrix, projected starting from the classical displacement-based discrete gradient B defined by Eqs. (14) and (15):

$$
\dot{\bar{\varepsilon}}(x, t)=\overline{\mathbf{B}}(x) \cdot \dot{\mathbf{d}}(t)
$$

Substituting the expression of $\dot{\overline{\boldsymbol{\varepsilon}}}$ given by (19) into the variational principle (18), one obtains:

$$
\delta \dot{\mathbf{d}}^{T} \cdot\left(\int_{\Omega_{e}} \overline{\mathbf{B}}^{T} \cdot \boldsymbol{\sigma} d \Omega-\mathbf{f}^{e x t}\right)=0
$$

Since $\delta \dot{\mathbf{d}}$ can be chosen arbitrarily, the previous equation is equivalent to:

$$
\mathbf{f}^{\text {int }}=\mathbf{f}^{\text {ext }}
$$

with:

$$
\mathbf{f}^{i n t}=\int_{\Omega_{e}} \overline{\mathbf{B}}^{T} \cdot \boldsymbol{\sigma}(\dot{\overline{\boldsymbol{\varepsilon}}}) d \Omega
$$

One should note that, in the above equation, the stress $\boldsymbol{\sigma}$ is deduced from the assumed-strain rate through the constitutive law. For problems involving nonlinear materials, $\boldsymbol{\sigma}$ is usually a function of the time history of the assumed-strain field and other internal variables:

$$
\boldsymbol{\sigma}=\mathscr{F}(\dot{\bar{\varepsilon}}, \boldsymbol{\alpha}, \ldots)
$$

where $\alpha$ denotes the internal variables. The formulation thus obtained is valid for problems involving both geometrical and material nonlinearities, provided that one takes into account the full strain tensor. In the case of linear problems, one has:
$\boldsymbol{\sigma}=\mathbf{C} \cdot \overline{\boldsymbol{\varepsilon}}$

In the same way as for the SHB8PS element [31, 32], an improved plane-stress type constitutive law is adopted here for the SHB6 element, in order to enhance its immunity with regard to thickness locking. This specific law, which uncouples the response in terms of in-plane and transverse normal stress versus normal strain, is given by:

$$
\begin{gathered}
\mathbf{C}=\left[\begin{array}{cccccc}
\bar{\lambda}+2 \mu & \bar{\lambda} & 0 & 0 & 0 & 0 \\
\bar{\lambda} & \bar{\lambda}+2 \mu & 0 & 0 & 0 & 0 \\
0 & 0 & E & 0 & 0 & 0 \\
0 & 0 & 0 & \mu & 0 & 0 \\
0 & 0 & 0 & 0 & k \mu & 0 \\
0 & 0 & 0 & 0 & 0 & k \mu
\end{array}\right] \\
\mu=\frac{E}{2(1+v)} \quad \bar{\lambda}=\frac{E v}{1-v^{2}}
\end{gathered}
$$

where $E$ is Young's modulus, $v$ is Poisson's ratio, and $k=5 / 6$. Note that as usually adopted, these material properties are specified with respect to a local physical coordinate system, in which the $x-y$ plane corresponds to the element mid-plane defined by the $\zeta$-coordinate of the considered integration point. The choice of this constitutive matrix avoids the locking encountered with a full three-dimensional law. Moreover, unlike the commonly adopted plane-stress assumption, this modified stiffness matrix allows the deformation energy associated with the strains normal to the mean surface of the element to be taken into consideration.

In the linear case, the element internal forces are given by:

$$
\mathbf{f}^{i n t}=\mathbf{K}_{e} \cdot \mathbf{d}
$$

with:

$$
\mathbf{K}_{e}=\int_{\Omega_{e}} \overline{\mathbf{B}}^{T} \cdot \mathbf{C} \cdot \overline{\mathbf{B}} d \Omega
$$

In the standard displacement approach, the stiffness matrix simply reduces to:

$$
\mathbf{K}_{e}=\int_{\Omega_{e}} \mathbf{B}^{T} \cdot \mathbf{C} \cdot \mathbf{B} d \Omega
$$

where $\mathbf{B}$ is the classical discrete gradient matrix.

\subsection{Analysis of the hourglass modes}

Hourglass modes are zero-strain-energy kinematic modes, also referred to as spurious or singular patterns, which arise as a consequence of reduced integration. This pathology can be 
Table 1. Coordinates and weights of the Gauss points for the 2-point quadrature SHB6 version.

\begin{tabular}{c|c|c|c|c}
\hline & $\xi$ & $\eta$ & $\zeta$ & $\omega$ \\
\hline $\mathrm{P}(1)$ & $1 / 3$ & $1 / 3$ & $-1 / \sqrt{3}$ & 1 \\
$\mathrm{P}(2)$ & $1 / 3$ & $1 / 3$ & $1 / \sqrt{3}$ & 1 \\
\hline
\end{tabular}

elucidated by comparing the dimension of the kernel of the discrete stiffness operator with that of the exact continuous stiffness matrix. The subintegrated discrete gradient operator (i.e., associated with a set of $n_{\text {int }}$ integration points located in $\xi_{I}=\eta_{I}=\frac{1}{3}, \zeta_{I}, \quad I=1, \ldots, n_{\text {int }}$, see Table 1 for $n_{\text {int }}=2$ ) is given by Eq. (15) with $\alpha$ ranging from one to two. Recall that this reduced integration aims to increase computational efficiency and to avoid some shear locking phenomena in bending-dominated problems. Accordingly, the elastic stiffness is obtained by Gauss integration:

$$
\mathbf{K}_{e}=\int_{\Omega_{e}} \mathbf{B}^{T} \cdot \mathbf{C} \cdot \mathbf{B} d \Omega=\sum_{I=1}^{n_{\mathrm{in}}} \omega\left(\zeta_{I}\right) J\left(\zeta_{I}\right) \mathbf{B}^{T}\left(\zeta_{I}\right) \cdot \mathbf{C} \cdot \mathbf{B}\left(\zeta_{I}\right)
$$

where $J\left(\zeta_{I}\right)$ is the Jacobian of the transformation between the unit reference configuration and the current configuration of an arbitrary prism. Table 1 gives the coordinates and the associated weights of integration points, which represent the roots of the Gauss-Legendre polynomial, in the case of a twopoint quadrature element.

Let us now examine the kernel of the stiffness matrix obtained with this set of integration points. From Eq. (29), one can see that the analysis of the kernel of the subintegrated stiffness boils down to studying the rank of matrix B, provided the constitutive matrix $\mathbf{C}$ is nonsingular. In other words, it suffices to seek the displacement modes $\mathbf{d}$ with zero strain, i.e., those that verify at each Gauss point:

$$
\nabla_{s}(\mathbf{u})=\mathbf{B}\left(\zeta_{I}\right) \cdot \mathbf{d}=\mathbf{0}
$$

In order to identify the kernel of the discrete stiffness matrix, a basis for the vector space of the discretized displacements is built. Note that, in general, reduced integration is shown to diminish the rank of the discrete stiffness. A detailed investigation of hourglass modes is given in Appendix B; hereafter, only the main results are reported. Using expression (15) for the discrete gradient operator computed at the integration points and making use of the orthogonality relations (10) and (16), the kernel of the stiffness matrix can be explicitly derived. This naturally reveals the six rigid body modes of which consists the kernel of a fully integrated stiffness:

$$
\left(\begin{array}{l}
\mathbf{s} \\
\mathbf{0} \\
\mathbf{0}
\end{array}\right),\left(\begin{array}{r}
\mathbf{0} \\
\mathbf{s} \\
\mathbf{0}
\end{array}\right),\left(\begin{array}{r}
\mathbf{0} \\
\mathbf{0} \\
\mathbf{s}
\end{array}\right),\left(\begin{array}{r}
\mathbf{y} \\
-\mathbf{x} \\
\mathbf{0}
\end{array}\right),\left(\begin{array}{r}
\mathbf{z} \\
\mathbf{0} \\
-\mathbf{x}
\end{array}\right),\left(\begin{array}{r}
\mathbf{0} \\
\mathbf{z} \\
-\mathbf{y}
\end{array}\right) .
$$

The first three 18-component column vectors correspond to the translations along the $O x, O y$, and $O z$ axes, respectively. The three remaining vectors refer to the rotations about the $O z, O y$, and $O x$ axes, respectively. On the whole, the kernel of a fully integrated stiffness matrix is of dimension six and only reduces to the above six rigid body modes.

Unlike the SHB8PS element (see Ref. [31]), which comprises six hourglass modes and requires stabilization, no zeroenergy modes are revealed from the hourglass analysis of the SHB6 element (see Appendix B for further details).

\subsection{Assumed-strain projection for locking treatment}

The discrete gradient operator $\mathbf{B}$ has to be projected onto an appropriate subspace in order to eliminate different locking phenomena; the projected operator will be denoted $\overline{\mathbf{B}}$. This assumed-strain method is consistent, from a variational perspective, with the $\mathrm{Hu}-\mathrm{Washizu}$ principle as long as the stress interpolation is appropriately chosen (see Simo and Hughes [59]). However, this variational justification of the assumedstrain method does not provide a general, systematic way to derive adequate assumed-strain fields, and a specific analysis of locking must be done for each new element developed based on this approach.

In this paper, we propose a projection scheme that is both effective and simple (see Ref. [45] for further details). The starting point consists of decomposing the discrete gradient operator $\mathbf{B}$ into two parts as follows:

$$
\mathbf{B}=\mathbf{B}_{1}+\mathbf{B}_{2}
$$

The first part, $\mathbf{B}_{1}$, contains the gradients in the element mid-plane (membrane terms of the deformation) as well as the normal strains, whereas the second part, $\mathbf{B}_{2}$, incorporates the gradients associated with the transverse shear strains:

$$
\begin{aligned}
\mathbf{B}_{1} & =\left[\begin{array}{ccc}
\mathbf{b}_{x}^{T}+h_{\alpha, x} \boldsymbol{\gamma}_{\alpha}^{T} & \mathbf{0} & \mathbf{0} \\
\mathbf{0} & \mathbf{b}_{y}^{T}+h_{\alpha, y} \boldsymbol{\gamma}_{\alpha}^{T} & \mathbf{0} \\
\mathbf{0} & \mathbf{0} & \mathbf{b}_{z}^{T}+h_{\alpha, z} \boldsymbol{\gamma}_{\alpha}^{T} \\
\mathbf{b}_{y}^{T}+h_{\alpha, y} \boldsymbol{\gamma}_{\alpha}^{T} & \mathbf{b}_{x}^{T}+h_{\alpha, x} \boldsymbol{\gamma}_{\alpha}^{T} & \mathbf{0} \\
\mathbf{0} & \mathbf{0} & \mathbf{0} \\
\mathbf{0} & \mathbf{0} & \mathbf{0}
\end{array}\right] \\
\mathbf{B}_{2} & =\left[\begin{array}{ccc}
\mathbf{0} & \mathbf{0} & \mathbf{0} \\
\mathbf{0} & \mathbf{0} & \mathbf{0} \\
\mathbf{0} & \mathbf{0} & \mathbf{0} \\
\mathbf{0} & \mathbf{0} & \mathbf{0} \\
\mathbf{b}_{z}^{T}+h_{\alpha, z} \gamma_{\alpha}^{T} & \mathbf{0} & \mathbf{b}_{z}^{T}+h_{\alpha, z} \boldsymbol{\gamma}_{\alpha}^{T}+h_{\alpha, x} \boldsymbol{b}_{\alpha}^{T}
\end{array}\right] .
\end{aligned}
$$

It has been observed, from numerical experiments, that the main locking mechanisms in the SHB6 element come from the transverse shears. Therefore, we choose an integration 
scheme that enables us to reduce the associated fraction in the total strain energy. In order to do this, the $\mathbf{B}_{2}$ matrix is projected as follows:

$$
\overline{\mathbf{B}}_{2}=\varepsilon \mathbf{B}_{2}
$$

where $\varepsilon$ is a shear scaling factor. By introducing the additive decomposition (32) of the B operator into Eq. (29) and making use of projection (35), the stiffness matrix becomes:

$$
\begin{aligned}
\mathbf{K}_{e} & =\int_{\Omega_{e}} \mathbf{B}_{1}^{T} \cdot \mathbf{C} \cdot \mathbf{B}_{1} d \Omega+\int_{\Omega_{e}} \mathbf{B}_{1}^{T} \cdot \mathbf{C} \cdot \overline{\mathbf{B}}_{2} d \Omega \\
& +\int_{\Omega_{e}} \overline{\mathbf{B}}_{2}^{T} \cdot \mathbf{C} \cdot \mathbf{B}_{1} d \Omega+\int_{\Omega_{e}} \overline{\mathbf{B}}_{2}^{T} \cdot \mathbf{C} \cdot \overline{\mathbf{B}}_{2} d \Omega
\end{aligned}
$$

which can be simply written as:

$$
\mathbf{K}_{e}=\mathbf{K}_{1}+\mathbf{K}_{2}
$$

The first term, $\mathbf{K}_{1}$, which is not affected by projection, is evaluated at the Gauss points as defined above:

$$
\mathbf{K}_{1}=\int_{\Omega_{e}} \mathbf{B}_{1}^{T} \cdot \mathbf{C} \cdot \mathbf{B}_{1} d \Omega=\sum_{I=1}^{n_{\text {int }}} \omega\left(\zeta_{I}\right) J\left(\zeta_{I}\right) \mathbf{B}_{1}^{T}\left(\zeta_{I}\right) \cdot \mathbf{C} \cdot \mathbf{B}_{1}\left(\zeta_{I}\right)
$$

The second term, $\mathbf{K}_{2}$, embodies all the projection and reads

$$
\mathbf{K}_{2}=\int_{\Omega_{e}} \mathbf{B}_{1}^{T} \cdot \mathbf{C} \cdot \overline{\mathbf{B}}_{2} d \Omega+\int_{\Omega_{e}} \overline{\mathbf{B}}_{2}^{T} \cdot \mathbf{C} \cdot \mathbf{B}_{1} d \Omega+\int_{\Omega_{e}} \overline{\mathbf{B}}_{2}^{T} \cdot \mathbf{C} \cdot \overline{\mathbf{B}}_{2} d \Omega
$$

The particular choice of additive decomposition, Eqs. (33) and (34), together with projection (35), yields a simplified form for the second part of the stiffness matrix, $\mathbf{K}_{2}$. Indeed, with these choices the first two terms, i.e. cross-terms, in the right-hand side of Eq. (39) vanish, and matrix $\mathbf{K}_{2}$ simply reduces to:

$$
\mathbf{K}_{2}=\int_{\Omega_{e}} \overline{\mathbf{B}}_{2}^{T} \cdot \mathbf{C} \cdot \overline{\mathbf{B}}_{2} d \Omega
$$

The identification of the shear scaling factor $\varepsilon$ in Eq. (35) has been carried out through numerical experiments, and the selected value for this parameter is found to be one half. This value is motivated by extensive testing on a variety of popular test problems, and it leads to reasonably good behavior for the element in most of the benchmark problems that have been tested.

\section{Numerical tests}

In order to evaluate this new assumed-strain version of the SHB6 element, it was tested on a series of linear elastic benchmark problems. For each test case, the obtained results were compared, on the one hand, to the reference solution found in the literature and, on the other hand, to the solutions given by both the standard three-dimensional six-node prism element PRI6 and the unmodified SHB6 element (i.e., without the assumed-strain projection). Note that the PRI6 is the wellknown linear solid element, which has a prismatic 3D shape, six nodes with three displacement degrees of freedom per node, linear interpolation functions, and $3 \times 2$ integration points. In all of the numerical tests, a single layer of elements was used through the thickness, along with only two integration points at the element level. Note also that in all of the tables reporting the convergence results, the meshes are indicated by the number of subdivisions in each direction (e.g., length, width), and the total number of elements is then doubled, since each rectangle is divided into two triangles. For example, the nomenclature $\left(N_{1} \times N_{2} \times 1\right) \times 2$ indicates $N_{1}$ elements in the length direction and $N_{2}$ elements in the width direction, while the total number of prisms is twice their product. In the following, the assumed-strain projected version of the SHB6 element will be denoted SHB6 ${ }^{\text {bar }}$.

The results given hereafter include a set of representative popular benchmark problems commonly used to test the performance of finite elements. These selected numerical examples are linear elastic problems and are mostly intended to assess the performance of the element in bending-dominated problems or to check its aspect-ratio limits. Examples of meshes containing both SHB6 ${ }^{\text {bar }}$ and SHB8PS elements demonstrate the advantage of using such combinations of hexahedral and prismatic solid-shell elements. The element is then tested in modal analysis (vibration eigenfrequencies of a clamped beam), and finally a buckling analysis is undertaken in order to evaluate its capabilities in solving elastic stability problems.

\subsection{Beam bending test}

This test problem enables one to evaluate the behavior of the element in pure bending. Indeed, in this test case, bending dominates over shearing.

The right-hand side of the beam is subjected to a shear load of resultant $P=4$; this loading is distributed among the end nodes. The left edge of the beam is fully clamped, as shown in Fig. 2(a).

The geometric, material, and loading data for the problem are given in Table 2 .

The reference deflection $w_{\text {ref }}$ of the tip point $\mathrm{A}$ is obtained analytically:

$$
w_{\text {ref }}=\frac{P L^{3}}{3 E I}, \quad \text { where: } \quad I=\frac{l t^{3}}{12} .
$$

The results in terms of convergence towards the reference solution are summarized in Table 3, which gives the displacement of the tip point $\mathrm{A}$ in the $z$-direction. One should 
Table 2. Geometric, material, and loading data for the beam bending problem.

\begin{tabular}{c|c|c}
\hline Length & $L$ & 50 \\
\hline Width & $l$ & 4 \\
\hline Thickness & $t$ & 1 \\
\hline Young's modulus & $E$ & $68.25 \times 10^{6}$ \\
\hline Poisson's ratio & $v$ & 0.3 \\
\hline Applied load & $P$ & 4 \\
\hline
\end{tabular}

Table 3. Normalized displacements in the $z$-direction at point A for the bending beam problem.

\begin{tabular}{c|c|c|c}
\hline \multirow{2}{*}{ Mesh layout } & PRI6 & SHB6 & SHB6 $^{\text {bar }}$ \\
\cline { 2 - 4 } & $w / w_{\text {ref }}$ & $w / w_{\text {ref }}$ & $w / w_{\text {ref }}$ \\
\hline$(6 \times 2 \times 1) \times 2$ & 0.037 & 0.121 & 0.382 \\
$(12 \times 2 \times 1) \times 2$ & 0.132 & 0.390 & 0.724 \\
$(24 \times 4 \times 1) \times 2$ & 0.356 & 0.662 & 0.899 \\
$(40 \times 4 \times 1) \times 2$ & 0.570 & 0.840 & 0.959 \\
$(50 \times 4 \times 1) \times 2$ & 0.649 & 0.887 & 0.972 \\
\hline
\end{tabular}

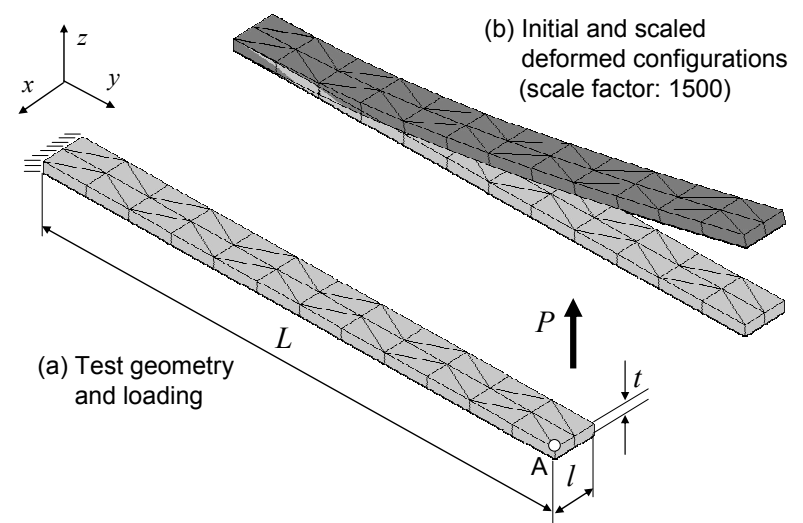

Fig. 2. Geometry, loading, and boundary conditions for the beam bending test problem: an example of a $(12 \times 2 \times 1) \times 2$ mesh.

note that the assumed-strain SHB $6^{\text {bar }}$ element converges faster than the SHB6 element without projection and the PRI6 prismatic element.

\subsection{Aspect-ratio limits for the element}

This test is intended to evaluate the aspect ratio limits of the element in a clamped beam in bending. The length and the width of the beam are fixed at $L=100, l=10$; while the thickness $t$ is a varying parameter. The elastic properties are $E$ $=68.25 \times 10^{6}$ and $v=0.3$. A bending load, $P=4$, is applied at the free edge of the beam, and the results are compared with the analytical solutions given by beam theory.

A fixed mesh of 20 SHB6 $^{\text {bar }}$ elements and a single element through the thickness is used; Fig. 3 illustrates this $(10 \times 1 \times 1) \times 2$ mesh for $t=1$. In this regular mesh, each element has a triangular basis (i.e., a $10 \times 10$ rectangular triangle), in which $l=$ 10 is the side of the triangle and $r=l / t$ is the varying as-

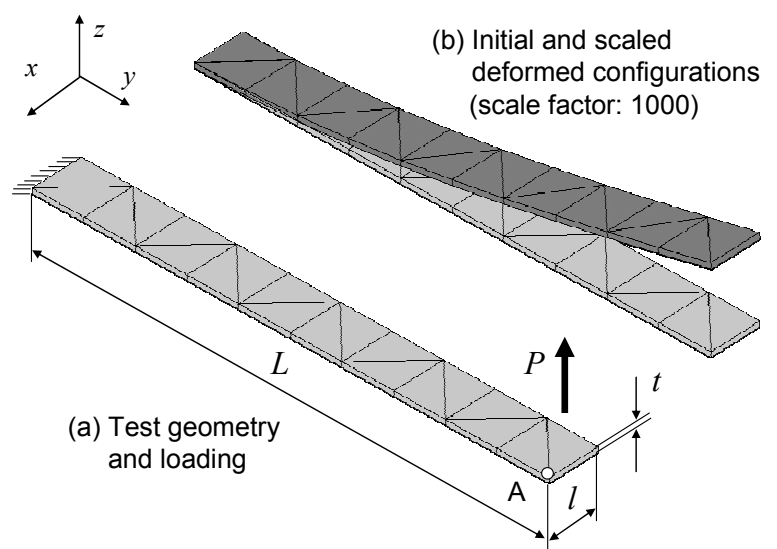

Fig. 3. Geometry, loading, and boundary conditions for the test of aspect-ratio limits: an example of a $(10 \times 1 \times 1) \times 2$ mesh with $t=1$.

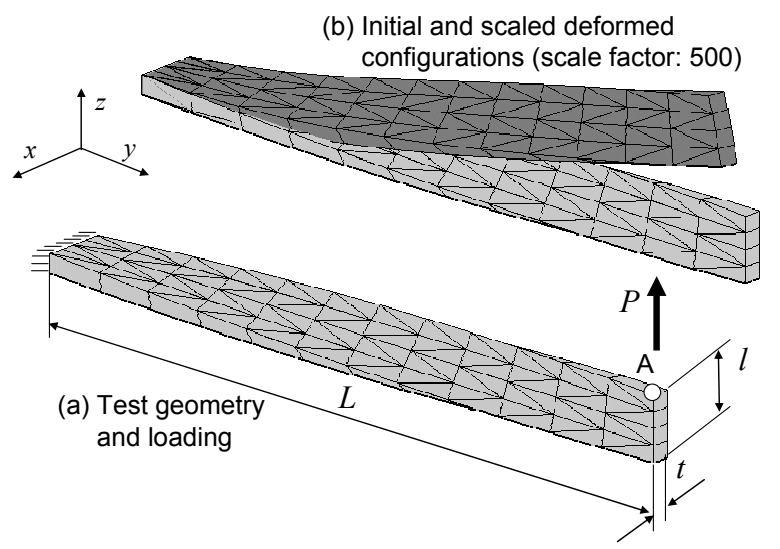

Fig. 4. Geometry, loading, and boundary conditions for the twisted beam test problem: an example of a $(12 \times 4 \times 1) \times 2$ mesh.

pect-ratio.

The investigation of the behavior of the element in the limit of high aspect ratios revealed that meshes with an aspect ratio $r=l / t$ between 0.5 and 20 provide deflections that are within $10 \%$ of the theoretical results. One should note that, unlike the SHB8PS element, for which the aspect-ratio limit was shown to be about 400 in this test problem, the SHB6 ${ }^{\text {bar }}$ element does not allow high aspect ratios.

\subsection{Twisted cantilever beam}

This test was introduced by MacNeal and Harder [60] and has been extensively used to test the performance of finite elements in cases of warped configurations. It is now considered as a reference shell test (see Batoz and Dhatt [61]). In the test, the initial beam geometry is twisted at an angle of $90^{\circ}$ between its two ends. This distorts the elements and thus increases the severity of the test.

Fig. 4 shows the geometry of the twisted beam, the boundary conditions, and the applied loading. The left end of this 
Table 4. Geometric, material, and loading data for the twisted beam problem.

\begin{tabular}{c|c|c}
\hline Length & $L$ & 12 \\
\hline Width & $l$ & 1.1 \\
\hline Thickness & $t$ & 0.32 \\
\hline Young's modulus & $E$ & $29 \times 10^{6}$ \\
\hline Poisson's ratio & $v$ & 0.22 \\
\hline Applied load & $P$ & 1 \\
\hline
\end{tabular}

Table 5. Normalized displacements in the $z$-direction at point $\mathrm{A}$ for the twisted beam problem.

\begin{tabular}{c|c|c|c}
\hline \multirow{2}{*}{ Mesh layout } & PRI6 & SHB6 & SHB6 $^{\text {bar }}$ \\
\cline { 2 - 4 } & $w / w_{\text {ref }}$ & $w / w_{\text {ref }}$ & $w / w_{\text {ref }}$ \\
\hline$(6 \times 2 \times 1) \times 2$ & 0.061 & 0.234 & 0.496 \\
$(12 \times 4 \times 1) \times 2$ & 0.202 & 0.470 & 0.784 \\
$(24 \times 4 \times 1) \times 2$ & 0.485 & 0.779 & 0.935 \\
$(36 \times 8 \times 1) \times 2$ & 0.489 & 0.875 & 0.972 \\
$(48 \times 8 \times 1) \times 2$ & 0.764 & 0.926 & 0.985 \\
\hline
\end{tabular}

beam is clamped, while a unit in-plane shear load, $P=1$, is applied at its right end in the $z$-direction. The geometric and material parameters for this problem are listed in Table 4.

The reference tip displacement in the loading direction $\mathrm{Oz}$ is given in Ref. [60]. For this in-plane loading $P$, the vertical displacement at point A (see Fig. 4) is expected to be equal to $w_{\text {ref }}=5.424 \times 10^{-3}$.

Table 5 shows the results for this test problem in terms of convergence as a function of the refinement of the mesh. The normalized displacements at point $\mathrm{A}$ in the loading direction $\mathrm{Oz}$ are reported for three elements, using different mesh densities and only one element through the thickness. Again, one can observe that element SHB6 ${ }^{\text {bar }}$ converges faster than elements SHB6 and PRI6.

\subsection{In-plane bending and shearing test}

This test case enables one to evaluate the behavior of the element submitted to in-plane transverse shearing and bending.

The loading consists of a resultant shear force $P$ applied to the right edge of the beam, leading to a parabolic stress distribution along the height and constant stress through the thickness. Since the beam is loaded in its plane, the evaluation concerns the membrane behavior of the element, which behaves essentially like a plane constant-stress triangle. Fully clamped boundary conditions are prescribed on the left edge of the plate (see Fig. 5).

The geometric, material, and loading data for this test problem are given in Table 6 .

The reference deflection of point A in the $O y$ direction was calculated analytically using the method of Airy's stress functions for plane-stress problems; the obtained reference value was $w_{\text {ref }}=-0.3573$. The convergence results are reported in Table 7. In this case, the three elements produced quasi-
Table 6. Geometric, material, and loading data for the shear-loaded beam problem.

\begin{tabular}{c|c|c}
\hline Length & $L$ & 48 \\
\hline Height & $H$ & 12 \\
\hline Thickness & $t$ & 1 \\
\hline Young's modulus & $E$ & $3 \times 10^{4}$ \\
\hline Poisson's ratio & $v$ & 0.25 \\
\hline Applied load & $P$ & 40 \\
\hline
\end{tabular}

Table 7. Normalized deflections in the $y$-direction at point A for the shear-loaded beam problem.

\begin{tabular}{c|c|c|c}
\hline \multirow{2}{*}{ Mesh layout } & PRI6 & SHB6 & SHB6 $^{\text {bar }}$ \\
\cline { 2 - 4 } & $w / w_{\text {ref }}$ & $w / w_{\text {ref }}$ & $w / w_{\text {ref }}$ \\
\hline$(6 \times 4 \times 1) \times 2$ & 0.594 & 0.605 & 0.620 \\
$(12 \times 4 \times 1) \times 2$ & 0.775 & 0.789 & 0.808 \\
$(24 \times 4 \times 1) \times 2$ & 0.840 & 0.854 & 0.866 \\
$(16 \times 16 \times 1) \times 2$ & 0.913 & 0.919 & 0.923 \\
$(32 \times 16 \times 1) \times 2$ & 0.966 & 0.970 & 0.976 \\
\hline
\end{tabular}

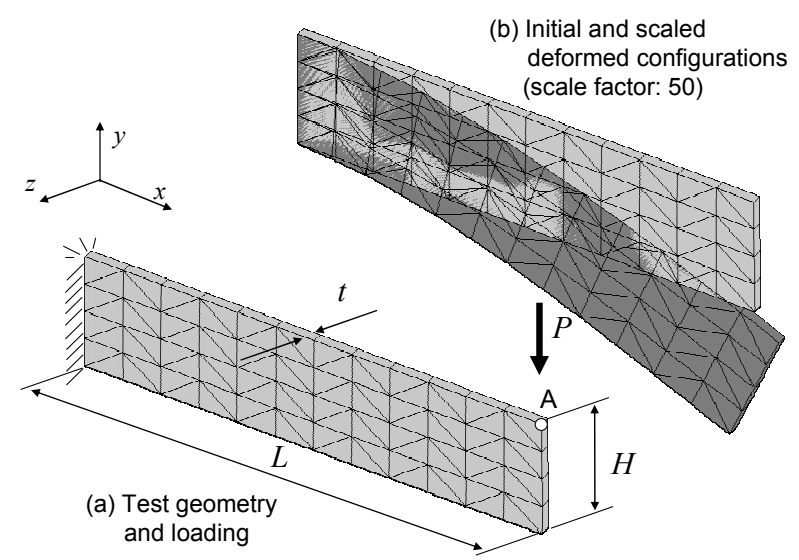

Fig. 5. Geometry, loading, and boundary conditions for the sheared beam test problem: an example of a $(12 \times 4 \times 1) \times 2$ mesh.

identical results, as expected.

\subsection{Circular plate subjected to a point load}

This test problem of a clamped circular plate under a concentrated central load allows the performance of the element in bending and shearing to be assessed.

Since the geometry, boundary conditions, and loading are symmetric, only one quarter of the plate is modeled using a $(3 \times(N \times N \times 1)) \times 2$ mesh nomenclature. Indeed, this quarter of the plate is divided into three zones containing $(N \times N \times 1) \times 2$ elements each (see Fig. 6 for an example of a $(3 \times(4 \times 4 \times 1)) \times 2$ mesh). The plate is subjected to a point load, $P=1$, at the center, and is clamped on its edge. The geometric and material properties for this problem are described in Table 8. The reference central deflection in the $O z$ direction is $w_{\text {ref }}=$ 
Table 8. Geometric, material, and loading data for the clamped circular plate problem.

\begin{tabular}{c|c|c}
\hline Radius & $R$ & 10 \\
\hline Thickness & $t$ & 0.5 \\
\hline Young's modulus & $E$ & $10^{7}$ \\
\hline Poisson's ratio & $v$ & 0.25 \\
\hline Applied load & $P$ & 1
\end{tabular}

Table 9. Normalized deflections in the $z$-direction at the load point A for the circular plate problem.

\begin{tabular}{c|c|c|c}
\hline \multirow{2}{*}{ Mesh layout } & PRI6 & SHB6 & SHB6 $^{\text {bar }}$ \\
\cline { 2 - 4 } & $w / w_{\text {ref }}$ & $w / w_{\text {ref }}$ & $w / w_{\text {ref }}$ \\
\hline$(3 \times(2 \times 2 \times 1)) \times 2$ & 0.162 & 0.422 & 0.692 \\
$(3 \times(4 \times 4 \times 1)) \times 2$ & 0.370 & 0.638 & 0.876 \\
$(3 \times(6 \times 6 \times 1)) \times 2$ & 0.522 & 0.753 & 0.942 \\
$(3 \times(8 \times 8 \times 1)) \times 2$ & 0.624 & 0.820 & 0.974 \\
$(3 \times(9 \times 9 \times 1)) \times 2$ & 0.686 & 0.843 & 0.996 \\
\hline
\end{tabular}

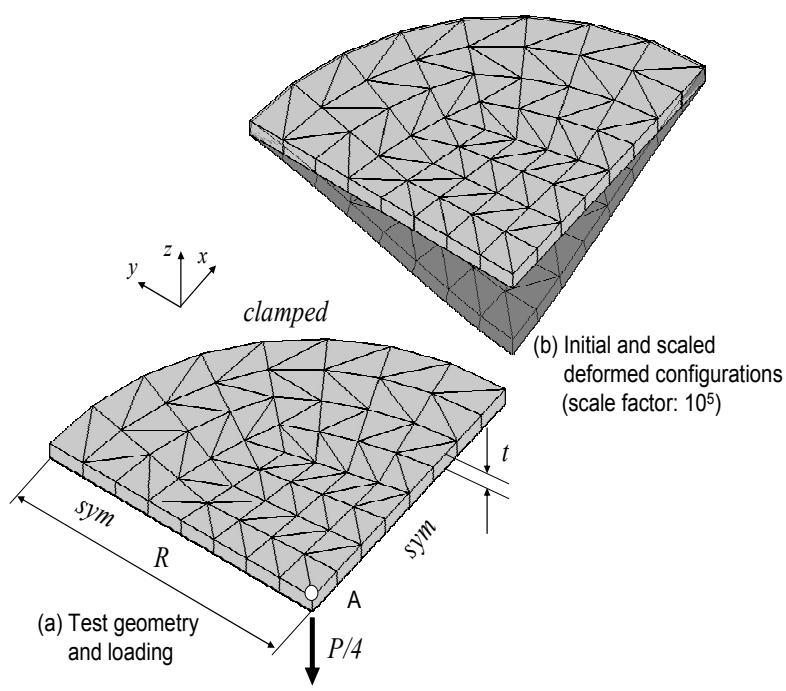

Fig. 6. Geometry, loading, and boundary conditions for the circular plate test problem: an example of a $(3 \times(4 \times 4 \times 1)) \times 2$ mesh.

\section{$-2.65736 \times 10^{-5}$.}

The normalized deflections of the load point A in the $O z$ direction are reported in Table 9 in terms of mesh refinement. As can be seen in this Table, element SHB6 ${ }^{\text {bar }}$ converges towards the reference solution better than the two other elements SHB6 and PRI6.

\subsection{Pinched cylindrical shell with end diaphragms}

A cylindrical shell loaded with a pair of concentrated vertical forces on its middle section is considered here. Both ends of the cylinder are covered with rigid diaphragms that allow displacements only in the axial direction (see Fig. 7). This test has been treated by many authors, including Belytschko et al.
Table 10. Geometric, material, and loading data for the pinched cylindrical shell.

\begin{tabular}{c|c|c}
\hline Length & $L$ & 600 \\
\hline Radius & $R$ & 300 \\
\hline Thickness & $t$ & 3 \\
\hline Young's modulus & $E$ & $3 \times 10^{6}$ \\
\hline Poisson's ratio & $v$ & 0.3 \\
\hline Applied load & $P$ & 1 \\
\hline
\end{tabular}

Table 11. Normalized deflections in the $z$-direction at point $\mathrm{A}$ for the pinched cylindrical shell.

\begin{tabular}{c|c|c|c}
\hline \multirow{2}{*}{ Mesh layout } & PRI6 & SHB6 & SHB6 $^{\text {bar }}$ \\
\cline { 2 - 4 } & $w / w_{\text {ref }}$ & $w / w_{\text {ref }}$ & $w / w_{\text {ref }}$ \\
\hline$(10 \times 10 \times 1) \times 2$ & 0.062 & 0.099 & 0.252 \\
$(30 \times 30 \times 1) \times 2$ & 0.219 & 0.349 & 0.699 \\
$(50 \times 50 \times 1) \times 2$ & 0.370 & 0.548 & 0.872 \\
$(70 \times 70 \times 1) \times 2$ & 0.490 & 0.688 & 0.947 \\
$(90 \times 90 \times 1) \times 2$ & 0.580 & 0.786 & 0.987 \\
\hline
\end{tabular}

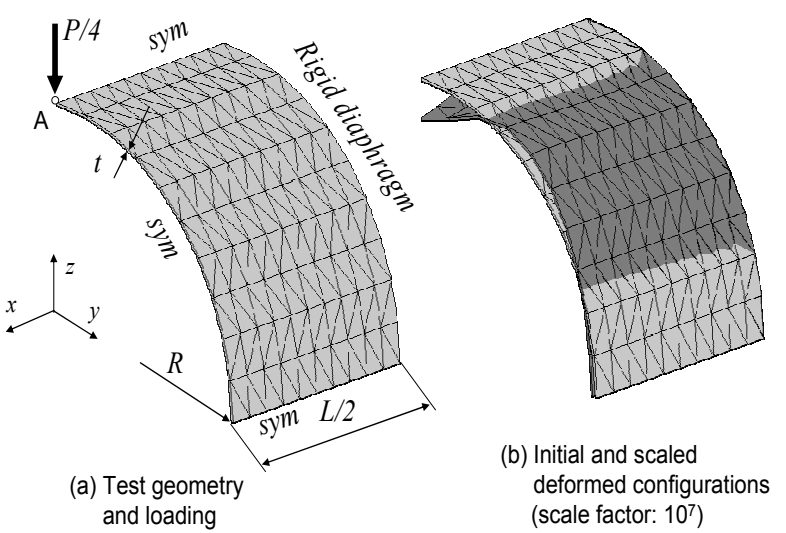

Fig. 7. Geometry, loading, and boundary conditions for the pinched cylinder test problem: an example of a $(10 \times 10 \times 1) \times 2$ mesh.

[62] and Chen and $\mathrm{Wu}$ [35]. It is considered as a selective test problem since they have shown that shear locking is more severe than membrane locking. The geometric and material parameters for this problem are reported in Table 10.

Due to the symmetry of the problem, only one eighth of the cylinder is modeled using different $(N \times N \times 1) \times 2$ meshes; an example of a $(10 \times 10 \times 1) \times 2$ mesh is shown in Fig. 7 .

The displacements at the load point $\mathrm{A}$ in the vertical $\mathrm{Oz}$ direction are normalized with respect to the reference solution $w_{\text {ref }}=-0.18248 \times 10^{-4}$ and are reported in Table 11 .

The convergence results shown in Table 11 reveal that element SHB6 ${ }^{\text {bar }}$ performs much better than both the former version without projection of SHB6 and the standard threedimensional six-node prism element PRI6.

\subsection{Pinched hemispherical shell}

This test problem, which is often used to assess the three- 
Table 12. Geometric, material, and loading data for the pinched hemispherical shell.

\begin{tabular}{c|c|c}
\hline Radius & $R$ & 10 \\
\hline Thickness & $t$ & 0.04 \\
\hline Young's modulus & $E$ & $6.825 \times 10^{7}$ \\
\hline Poisson's ratio & $v$ & 0.3 \\
\hline Applied load & $F$ & 1
\end{tabular}

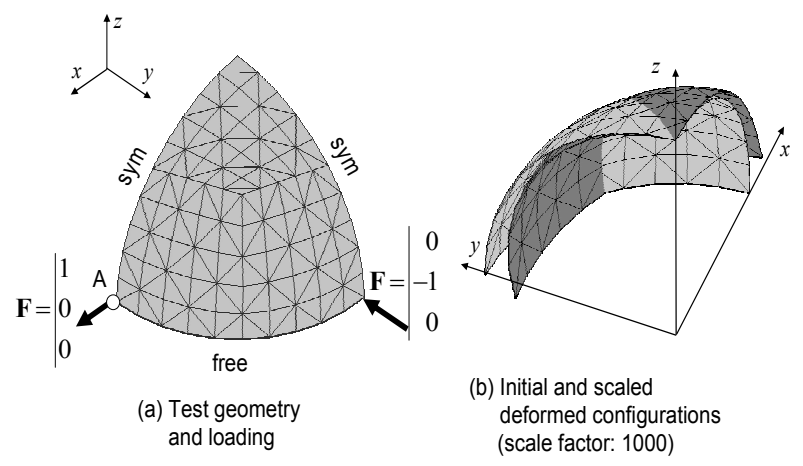

Fig. 8. Geometry, loading, and boundary conditions for the hemispherical shell test problem: an example of a $(3 \times(5 \times 5 \times 1)) \times 2$ mesh.

dimensional inextensional bending behavior of shells, has become very popular and has been adopted by many authors since it was proposed by MacNeal and Harder [60]. This test is known to be severe because the transverse shear and membrane locking phenomena are dominant and are further accentuated by the particular geometry of the problem (distorted, skewed elements). This problem was studied in detail by Belytschko et al. [62], who showed that since all the elements are incurved in this doubly-curved shell problem, the intensity of membrane and shear locking is increased. Fig. 8(a) shows the geometry, loading, and boundary conditions for this elastic thin shell problem $(R / t=250)$.

The geometric and material properties and the applied loading for this test problem are listed in Table 12.

Due to the symmetry of the problem (i.e., planes $(x z)$ and $(y z)$ ), only one quarter of the hemisphere is meshed using a single layer of elements through the thickness and with two unit loads along the directions $O x$ and $O y$. Except for the symmetry, the boundary conditions are free; nevertheless, the displacement of one point in the $z$-direction is fixed in order to prevent rigid body motions. According to the reference solution (MacNeal and Harder [60]), the displacement of point A along the $x$-direction is $w_{\text {ref }}=0.0924$ (see Fig. 8).

The convergence results are reported in Table 13 in terms of normalized displacements at point $\mathrm{A}$ in the $x$-direction versus the number of elements. The mesh nomenclature adopted for this test is similar to that used for the circular plate subjected to a point load (Section 3.5). This nomenclature consists of a $(3 \times(N \times N \times 1)) \times 2$ mesh, in which the quarter of the hemisphere is divided into three zones containing $(N \times N \times 1) \times 2$ elements each (see Fig. 8 for an example of a $(3 \times(5 \times 5 \times 1)) \times 2$ mesh).
Table 13. Normalized displacements in the $x$-direction at point A for the pinched hemispherical shell.

\begin{tabular}{c|c|c|c}
\hline \multirow{2}{*}{ Mesh layout } & PRI6 & SHB6 & SHB6 $^{\text {bar }}$ \\
\cline { 2 - 4 } & $w / w_{\text {ref }}$ & $w / w_{\text {ref }}$ & $w / w_{\text {ref }}$ \\
\hline$(3 \times(5 \times 5 \times 1)) \times 2$ & 0.001 & 0.006 & 0.017 \\
\hline$(3 \times(11 \times 11 \times 1)) \times 2$ & 0.005 & 0.013 & 0.046 \\
\hline$(3 \times(22 \times 22 \times 1)) \times 2$ & 0.022 & 0.059 & 0.195 \\
\hline$(3 \times(180 \times 180 \times 1)) \times 2$ & 0.549 & 0.695 & 0.917 \\
\hline$(3 \times(250 \times 250 \times 1)) \times 2$ & 0.696 & 0.810 & 0.957 \\
\hline
\end{tabular}

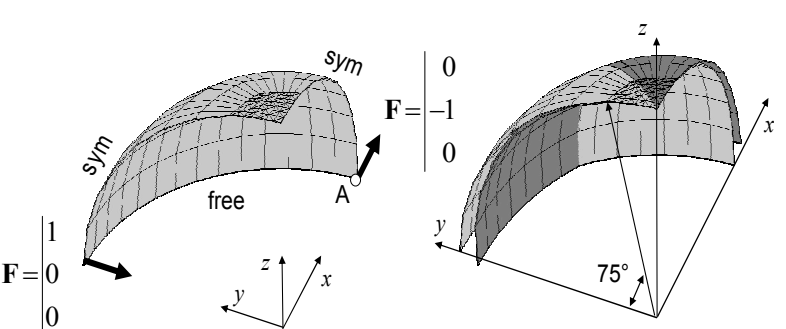

(a) Test geometry

(b) Initial and scaled and loading

(scale factor: 10)

Fig. 9. Pinched hemispherical shell problem with a mixture of prismatic and hexahedral elements: the SHB6 elements are located at the top, and the SHB8PS elements are arranged over an angle of $75^{\circ}$.

The new assumed-strain version SHB6 ${ }^{\text {bar }}$ is compared to the former version without projection, SHB6, and to the standard, six-node prism, full integration solid element, PRI6. Table 13 shows that even though the SHB6 ${ }^{\text {bar }}$ element converges very slowly towards the reference solution, it is still better than the former version without projection, SHB6, and is much better than the standard three-dimensional six-node prism element. The deflection is illustrated in Fig. 8(b), using a deformed configuration scaled with an amplification factor $10^{3}$, for a mesh with $(3 \times(5 \times 5 \times 1)) \times 2$ elements.

\subsection{Pinched hemispherical shell with a mixture of elements}

Since the primary aim in developing the prismatic solid-

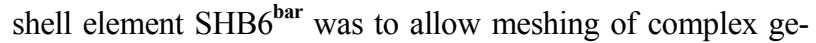
ometries that could not be meshed with solely hexahedral solid-shell elements like SHB8PS [31, 32], the previous test problem was reconsidered using a mixture of SHB6 and SHB8PS elements. In these mixed meshes, the SHB6 elements were located successively at the top (far away from the point of application of the loads, see Fig. 9) and at the three corners of the quarter-hemisphere (see Fig. 10). For the first case, the mesh domain spanned with SHB8PS elements was defined by an angle of $75^{\circ}$, as shown in Fig. 9(b). For the other cases, the SHB8PS elements were arranged over $60^{\circ}$, $75^{\circ}$, or $80^{\circ}$. Note also that in order to compare the performance of solid-shell elements to that of standard threedimensional elements, SHB6 elements were mixed with SHB8PS elements, and PRI6 elements were mixed with their 
Table 14. Normalized displacements in the $x$-direction at point A for the pinched hemispherical shell: mixed meshes in which SHB6 elements are located at the top.

\begin{tabular}{c|c|c|c}
\hline \multirow{2}{*}{ Number of elements } & $\begin{array}{c}\text { PRI6 }+ \\
\text { HEX8 }\end{array}$ & $\begin{array}{c}\text { SHB6 }+ \\
\text { SHB8PS }\end{array}$ & $\begin{array}{c}\text { SHB6 } \\
\text { SHB8r }+\end{array}$ \\
\cline { 2 - 4 } & $w / w_{\text {ref }}$ & $w / w_{\text {ref }}$ & $w / w_{\text {ref }}$ \\
\hline 36 & 0.001 & 0.703 & 0.785 \\
100 & 0.002 & 0.880 & 0.960 \\
156 & 0.004 & 0.929 & 0.983 \\
400 & 0.008 & 0.981 & 0.997 \\
\hline
\end{tabular}
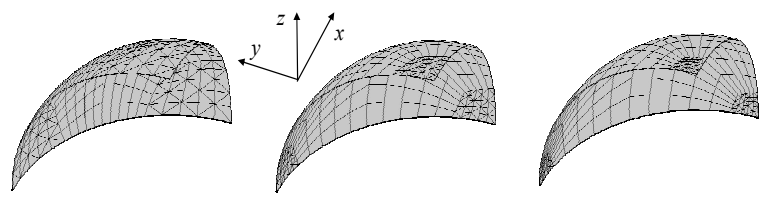

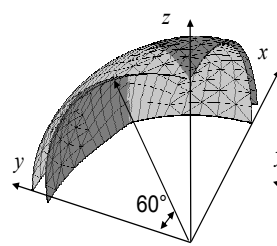

(deformed configuration scaled with a factor 50)

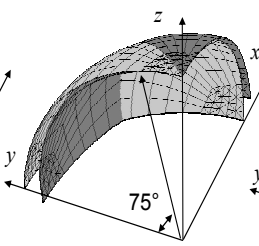

(deformed configuration scaled with a factor 20)

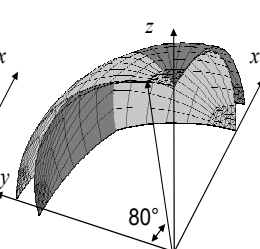

(deformed configuration scaled with a factor 20)
Fig. 10. Pinched hemispherical shell problem with a mixture of prismatic and hexahedral elements: the SHB6 elements are located at the three corners, and the SHB8PS elements are arranged over angles of $60^{\circ}, 75^{\circ}$, and $80^{\circ}$, respectively.

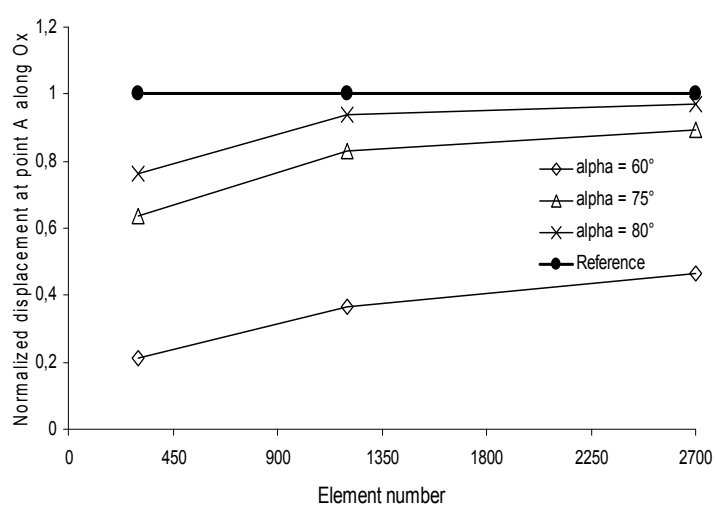

Fig. 11. Convergence curves for the pinched hemisphere test problem: the SHB6 elements are placed in the three corners, and the SHB8PS elements are arranged over angles of $60^{\circ}, 75^{\circ}$, and $80^{\circ}$, respectively.

three-dimensional counterpart HEX8, which are the standard, full integration eight-node hexahedral elements.

For the first case, where the SHB6 elements are placed at the top of the hemisphere (see Fig. 9), one can observe a convergence rate very similar to that of the SHB8PS element (see Table 14).

For the other cases (Fig. 10), the convergence as a function of mesh refinement is shown in Fig. 11. One can observe that
Table 15. Geometric and material properties for the test of a clamped beam in vibration.

\begin{tabular}{c|c|c}
\hline Length & $L$ & 1 \\
\hline Width & $b$ & 0.1 \\
\hline Thickness & $t$ & 0.01 \\
\hline Young's modulus & $E$ & $210 \times 10^{9}$ \\
\hline Poisson's ratio & $v$ & 0.3 \\
\hline Density & $\rho$ & 7800
\end{tabular}

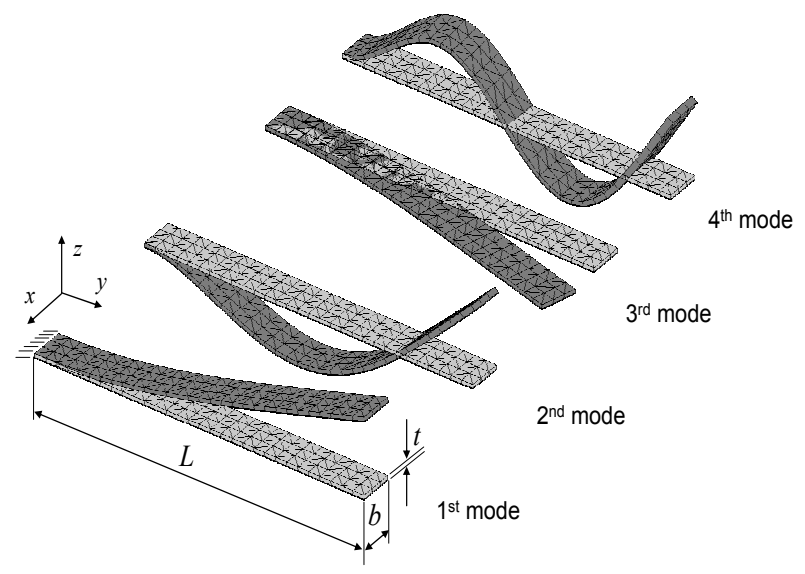

Fig. 12. Vibration test of a clamped beam: illustration of the first four eigenmodes obtained with a mesh consisting of $(30 \times 3 \times 1) \times 2 \mathrm{SHB}^{\text {bar }}$ elements.

the convergence is rather fast when the angle of the SHB8PS mesh is greater than or equal to $75^{\circ}$. However, when the SHB6 $^{\text {bar }}$ elements occupy a large portion of the domain, one reverts to the slow convergence of the SHB6 elements alone for this pinched hemispherical shell problem, as shown in the previous section.

\subsection{Vibration test: eigenfrequencies of a cantilever beam}

In this test problem, the $\mathrm{SHB}^{\text {bar }}$ element was tested in vibration using the same mass matrix as that of the standard three-dimensional six-node prismatic element. The first four eigenfrequencies of a clamped beam were investigated. The geometric and material properties of this test problem are given in Table 15.

In Tables 16-18, the results obtained with the three elements SHB6 ${ }^{\text {bar }}$, SHB6, and PRI6, for the same meshes, are compared to the analytical results given by beam theory. This comparison reveals that not only the $\mathrm{SHB}^{\text {bar }}{ }^{\text {is more accurate }}$ than SHB6 and PRI6, but also it converges much better with mesh refinement. Another interesting observation is that, for the third mode which involves a moderately thick structure (i.e. vibration in the $x-y$ plane), the SHB6 ${ }^{\text {bar }}$ element still behaves well. This demonstrates its capability of dealing with both thin and moderately thick structures, in contrast to solid elements like PRI6. The same analysis was then performed 
Table 16. Vibration eigenfrequencies for the clamped beam test problem: comparison of error percentages between analytical beam theory and $\mathrm{SHB}^{\text {bar }}$ results.

\begin{tabular}{c|c|c|c|c}
\hline \multirow{2}{*}{$\begin{array}{c}\text { Analytical } \\
\text { results }\end{array}$} & \multicolumn{4}{|c}{ Number of SHB6 ${ }^{\text {bar }}$ elements } \\
\cline { 2 - 5 } & $(30 \times 3 \times 1) \times 2$ & $(40 \times 4 \times 1) \times 2$ & $(80 \times 8 \times 1) \times 2$ & $(120 \times 12 \times 1) \times 2$ \\
\hline $\begin{array}{c}\text { Eigenfre- } \\
\text { quencies } \\
(\mathrm{Hz})\end{array}$ & Error (\%) & Error (\%) & Error (\%) & Error (\%) \\
\hline$f_{1}=8.4$ & 13.8 & 9.3 & 3.0 & 1.6 \\
\hline$f_{2}=52.5$ & 13.5 & 8.9 & 3.1 & 1.7 \\
\hline$f_{3}=83.8$ & 15.8 & 9.6 & 2.5 & 0.9 \\
\hline$f_{4}=147.1$ & 12.6 & 8.2 & 2.7 & 1.4 \\
\hline
\end{tabular}

Table 17. Vibration eigenfrequencies for the clamped beam test problem: comparison of error percentages between analytical beam theory and SHB6 results.

\begin{tabular}{c|c|c|c|c}
\hline \multirow{2}{*}{$\begin{array}{c}\text { Analytical } \\
\text { results }\end{array}$} & \multicolumn{4}{|c}{ Number of SHB6 elements } \\
\cline { 2 - 5 } & $(30 \times 3 \times 1) \times 2$ & $(40 \times 4 \times 1) \times 2$ & $(80 \times 8 \times 1) \times 2$ & $(120 \times 12 \times 1) \times 2$ \\
\hline $\begin{array}{c}\text { Eigenfre- } \\
\text { quencies } \\
(\mathrm{Hz})\end{array}$ & Error (\%) & Error (\%) & Error (\%) & Error (\%) \\
\hline$f_{1}=8.4$ & 46.4 & 32.1 & 10.5 & 5.3 \\
\hline$f_{2}=52.5$ & 46.3 & 31.5 & 10.7 & 5.5 \\
\hline$f_{3}=83.8$ & 15.9 & 9.6 & 2.5 & 0.8 \\
\hline$f_{4}=147.1$ & 45.4 & 31.1 & 10.6 & 5.4 \\
\hline
\end{tabular}

Table 18. Vibration eigenfrequencies for the clamped beam test problem: comparison of error percentages between analytical beam theory and PRI6 results.

\begin{tabular}{c|c|c|c|c}
\hline \multirow{2}{*}{$\begin{array}{c}\text { Analytical } \\
\text { results }\end{array}$} & \multicolumn{4}{|c}{ Number of PRI6 elements } \\
\cline { 2 - 5 } & $(30 \times 3 \times 1) \times 2$ & $(40 \times 4 \times 1) \times 2$ & $(80 \times 8 \times 1) \times 2$ & $(120 \times 12 \times 1) \times 2$ \\
\hline $\begin{array}{c}\text { Eigenfre- } \\
\text { quencies } \\
(\mathrm{Hz})\end{array}$ & Error (\%) & Error (\%) & Error (\%) & Error (\%) \\
\hline$f_{1}=8.4$ & 128.6 & 88.1 & 33.3 & 19.1 \\
\hline$f_{2}=52.5$ & 83.6 & 73.9 & 33.9 & 19.6 \\
\hline$f_{3}=83.8$ & 43.7 & 18.3 & 2.2 & 0.7 \\
\hline$f_{4}=147.1$ & 129.7 & 88.8 & 33.9 & 19.6 \\
\hline
\end{tabular}

Table 19. Vibration eigenfrequencies for the clamped beam test problem: comparison of error percentages between analytical beam theory and SHB8PS results.

\begin{tabular}{c|c|c|c|c}
\hline \multirow{2}{*}{$\begin{array}{c}\text { Analytical } \\
\text { results }\end{array}$} & \multicolumn{4}{|c}{ Number of SHB8PS elements } \\
\cline { 2 - 5 } & $(10 \times 2 \times 1)$ & $(20 \times 4 \times 1)$ & $(30 \times 6 \times 1)$ & $(40 \times 8 \times 1)$ \\
\hline $\begin{array}{c}\text { Eigenfre- } \\
\text { quencies } \\
(\mathrm{Hz})\end{array}$ & Error (\%) & Error $(\%)$ & Error (\%) & Error (\%) \\
\hline$f_{1}=8.4$ & 0.7 & 0.5 & 0.4 & 0.4 \\
\hline$f_{2}=52.5$ & 4.6 & 1.6 & 1.1 & 0.8 \\
\hline$f_{3}=83.8$ & 1.3 & 0.1 & 0.4 & 0.5 \\
\hline$f_{4}=147.1$ & 11.9 & 3.2 & 1.7 & 1.2 \\
\hline
\end{tabular}

Table 20. Geometric and material properties for the stiffened ring under external pressure.

\begin{tabular}{c|c|c}
\hline Frame spacing & $L_{s}$ & 0.6 \\
\hline Mean radius & $R$ & 2.488 \\
\hline Shell thickness & $e$ & 0.024 \\
\hline Web height & $h_{w}$ & 0.156 \\
\hline Flange width & $W_{f}$ & 0.120 \\
\hline Web thickness & $e_{w}$ & 0.010 \\
\hline Flange thickness & $e_{f}$ & 0.024 \\
\hline Young's modulus & $E$ & $200 \times 10^{9}$ \\
\hline Poisson's ratio & $v$ & 0.3 \\
\hline
\end{tabular}

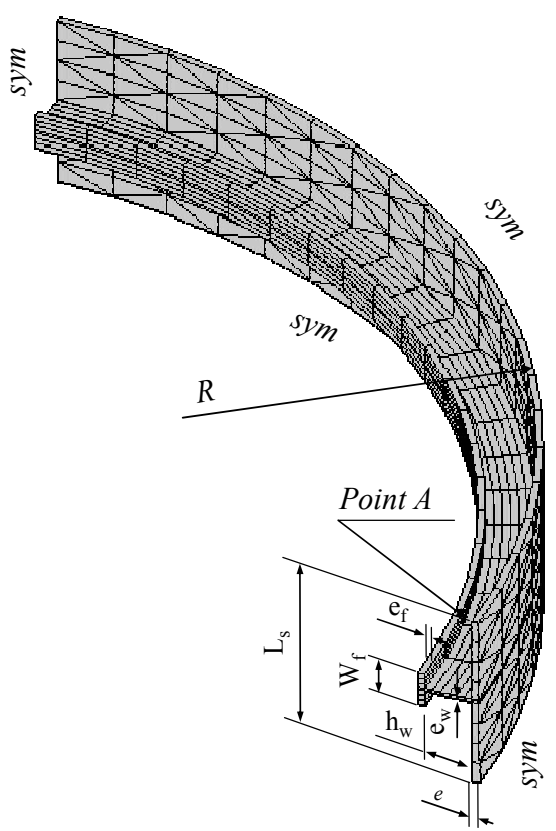

Fig. 13. Geometry and boundary conditions for a quarter of a single ring of the stiffened cylinder test problem: an example of a mixed mesh using 260 SHB8PS elements for the stiffener and 360 SHB6 $^{\text {bar }}$ elements for the main shell.

with the SHB8PS element, but with coarser meshes. Table 19 shows the convergence results. Once again, one can observe the high coarse-mesh accuracy of the SHB8PS element.

\subsection{Buckling analysis of a stiffened cylindrical ring}

In this last benchmark problem, the SHB6 elements were tested in the framework of elastic stability analysis. This problem also allows the verification of the formulation of the geometric stiffness matrix $\mathbf{K}_{\sigma}$ (see Appendix A.3). The test consists of a portion of a submarine hull subjected to external pressure, as illustrated in Fig. 13. The test was previously used in Ref. [63], while in Ref. [64], the submarine was modeled in its entirety. The following analysis only considers a single ring, as described in Fig. 13; the geometric and material data are 


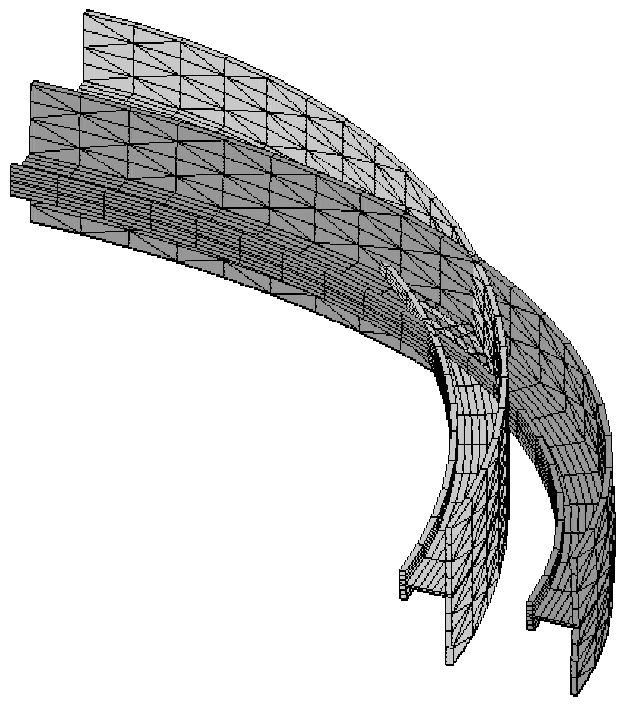

Fig. 14. Buckling mode for the stiffened cylinder test: quarter-ring consisting of 260 SHB8PS elements for the stiffener and 360 SHB6 $6^{\text {bar }}$ elements for the main shell.

reported in Table 20. Note that due to its symmetry, only one quarter of the ring is modeled and subjected to the corresponding symmetry boundary conditions (see Fig. 13). A linear buckling analysis was performed to determine the Euler critical pressure, based on the eigenvalue analysis of the global stiffness matrix.

In this linear buckling analysis, the Euler critical pressure is determined along with the corresponding buckling mode. This critical state is associated with the lowest pressure that makes the global stiffness matrix singular and is classically obtained by solving the following eigenvalue problem:

$$
\left(\mathbf{K}_{e}+\lambda_{c} \mathbf{K}_{\sigma}\right) \cdot \mathbf{X}_{c}=\mathbf{0}
$$

in which $\lambda_{c}$ is the critical buckling load and $\mathbf{X}_{c}$ is the associated buckling mode. The results were compared to those obtained with the SHB8PS element. This latter was first used to obtain a reference solution for the first critical load (i.e., dead load buckling pressure). A converged solution obtained with a fine mesh of 440 SHB8PS elements is $7.06 \times 10^{6}$, which is in good agreement with previously reported results [63, 64]. Note that the SHB8PS element was validated through a variety of elastic and elastic-plastic stability analyses (see, e.g., Refs. $[65,66])$, and could therefore be considered as accurate and reliable in buckling problems. As already discussed in Section 3.8, mixed meshes are again employed to emphasize the interest of combining hexahedral and prismatic elements in certain applications. These mixed meshes consist successively of 260 SHB8PS elements for the stiffener and 360 SHB6 ${ }^{\text {bar }}$ elements for the main shell, then 260 SHB8PS (stiffener) and 360 SHB6 (shell), and finally 260 HEX8 (stiffener) and 360 PRI6 (shell). The obtained results for the three elements thus mixed (SHB6 ${ }^{\text {bar }}$, SHB6, and PRI6) are, respec- tively, $7.04 \times 10^{6}, 7.20 \times 10^{6}$, and $7.61 \times 10^{6}$, which corresponds to error percentages of $0.3 \%, 2 \%$, and $7.8 \%$, respectively. These results confirm, once again, the advantage of mixing elements SHB8PS and SHB6 ${ }^{\text {bar }}$. The associated buckling mode is shown in Fig. 14.

\section{Discussion and conclusions}

The formulation of the SHB6 solid-shell element has been improved with regard to locking, and a new assumed-strain

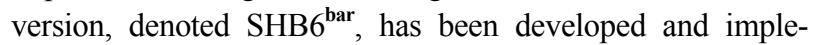
mented into the implicit, nonlinear finite element code ASTER. This resulting derivation has been evaluated on a variety of popular benchmark problems commonly used in the literature. The key idea of this development is the adequate combination of a reduced integration rule with the well-known assumed-strain method. An interesting feature of this approach is the convenient fully three-dimensional framework on which this solid-shell element is based (six-node prism with only three translational degrees of freedom per node).

In order to achieve an attractive, low-cost formulation, the computational efficiency of the element has been enhanced by adopting an in-plane one-point quadrature scheme. It has been shown that no zero-energy modes arise from this reduced integration, and thus no stabilization procedure is required. For the out-of-plane integration, two Gauss points have been chosen along a particular direction, designated as the "thickness". These choices, along with the use of a modified elastic constitutive law specifically aimed at reducing thickness locking, all contribute to a more accurate analysis of bendingdominated structural problems using only a single layer of elements along the thickness. Moreover, the projection technique adopted in the current formulation is intended to alleviate certain locking phenomena. Another important feature of this formulation is that it is valid for an arbitrary number, $n_{\text {int }} \geq 2$, of integration points along the thickness direction. The minimum number of two integration points in thickness direction is required in order to avoid spurious mechanisms associated with stiffness matrix rank deficiency. This feature proves to be useful for non linear analyses, in which the adequate number of integration points required to deal with elastic-plastic benchmark problems will be discussed and reported in a subsequent study.

As revealed by the benchmark problems, the $\mathrm{SHB} 6^{\text {bar }}$ element brings significant improvements compared to the standard three-dimensional six-node prismatic element denoted PRI6. The projection using the assumed-strain technique makes the quality of the element even better under combined bending and shearing. However, the convergence remains rather slow in the pinched hemispherical shell test, probably due to the fact that strains in linear triangular elements are constant. This type of element blends naturally with the eightnode hexahedral solid-shell element SHB8PS, which enables one to analyze any structural geometry quite easily. This is not the case when using hexahedral elements alone. Given the 
relatively slower convergence of the $\mathrm{SHB} 6^{\text {bar }}$ element, it is advisable to mesh with eight-node hexahedrons wherever possible, then to use $\mathrm{SHB} 6^{\text {bar }}$ elements only for the remainder of the structure.

\section{Acknowledgment}

The authors would like to thank EDF (the French Electricity Company) for its funding for this project, and for providing them with the finite element software ASTER. The financial support from CETIM (Technical Center for Mechanical Industries) is also gratefully acknowledged.

\section{References}

[1] S. Ahmad, Analysis of thick and thin shell structures by curved finite elements, Int. J. Numer. Methods Eng., 2 (1970) 419-451.

[2] T. J. R. Hughes and W. K. Liu, Nonlinear finite element analysis of shells: Part I. Three-dimensional shells, Comput. Methods Appl. Mech. Eng., 26 (2001) 331-362.

[3] K. J. Bathe and E. N. Dvorkin, Four-node plate bending element based on Mindlin/ Reissner plate theory and a mixed interpolation, Int. J. Numer. Methods Eng., 21 (1985) 367-383.

[4] K. J. Bathe and E. N. Dvorkin, Formulation of general shell elements - The use of mixed interpolation of tensorial components, Int. J. Numer. Methods Eng., 22 (1986) 697-722.

[5] J. C. Simo, D. D. Fox and M. S. Rifai, On a stress resultant geometrically exact shell model. Part III. Computational aspects of the nonlinear theory, Comput. Methods Appl. Mech. Eng., 79 (1990) 21-70.

[6] Y. Cheung and W. J. Chen, Refined hybrid method for plane isoparametric element using an orthogonal approach, Comput. Struct., 42 (1992) 683-694.

[7] E. Onate and J. Castro, Derivation of plate based on assumed shear strain fields, In: P. Ladevèze, O. C. Zienkiewicz, editors, New Advances in Computational Structures Mechanics, Amsterdam: Elsevier (1992) 237-288.

[8] P. Wriggers and F. Gruttmann, Thin shells with finite rotations formulated in Biot stress: theory and finite element formulation, Int. J. Numer. Methods Eng., 36 (1993) 20492071.

[9] R. Ayad, G. Dhatt and J. L. Batoz, A new hybrid-mixed variational approach for Reissner-Mindlin plates: The MiSP model, Int. J. Numer. Methods Eng., 42 (1998) 1149-1179.

[10] D. Chapelle and K. J. Bathe, Fundamental considerations for the finite element analysis of shell structures, Comput. Struct., 66 (1998) 19-36.

[11] Q. Zeng and A. Combescure, A new one point quadrature, general non-linear quadrilateral shell element with physical stabilization, Int. J. Numer. Methods Eng., 42 (1998) 13071338.

[12] H. Parisch, An investigation of a finite rotation four-node shell element, Int. J. Numer. Methods Eng., 31 (1991) 127-
150.

[13] N. Buechter, E. Ramm and D. Roehl, Three-dimensional extension of non-linear shell formulation based on the enhanced assumed strain concept, Int. J. Numer. Methods Eng., 37 (1994) 2551-2568.

[14] P. Betsch, F. Gruttmann and E. Stein, A 4-node finite shell element for the implementation of general hyperelastic 3Delasticity at finite strains, Comput. Methods Appl. Mech. Eng., 130 (1996) 57-79.

[15] M. Bischoff and E. Ramm, Shear deformable shell elements for large strains and rotations, Int. J. Numer. Methods Eng., 40 (1997) 4427-4449.

[16] B. Brank, J. Korelc and A. Ibrahimbegovic, Nonlinear shell problem formulation accounting for through-the-thickness stretching and its finite element implementation, Comput. Struct., 80 (2002) 699-717.

[17] R. P. R. Cardoso and J. W. Yoon, One point quadrature shell element with through-thickness stretch, Comput. Methods Appl. Mech. Eng., 194 (2005) 1161-1199.

[18] W. Graf, T. Y. Chang and A. F. Saleeb, On the numerical performance of three-dimensional thick shell elements using a hybrid/mixed formulation, Finite Elements Anal. Des., 2 (1986) 357-375.

[19] X. Xu and R. Cai, A new plate shell element of 16 nodes and 40 degrees of freedom by relative displacement method, Commun. Numer. Methods Eng., 9 (1993) 15-20.

[20] K. Y. Sze and A. Ghali, Hybrid hexahedral element for solids, plates, shells and beams by selective scaling, Int. J. Numer. Methods Eng., 36 (1993) 1519-1540.

[21] Y. H. Kim and S. W. Lee, A solid element formulation for large deflection analysis of composite shell structures, Comput. Struct., 30 (1993) 269-274.

[22] D. N. Buragohain and P. K. Ravichandran, Modified threedimensional finite element for general and composite shells, Comput. Struct., 51 (1994) 289-298.

[23] E. Domissy, Formulation et évaluation d'éléments finis volumiques modifiés pour l'analyse linéaire et non linéaire des coques, $\mathrm{PhD}$ thesis, UT Compiègne, France (1997).

[24] C. Cho, H. C. Park and S. W. Lee, Stability analysis using a geometrically nonlinear assumed strain solid shell element model, Finite Elements Anal. Des., 29 (1998) 121-135.

[25] R. Hauptmann and K. Schweizerhof, A systematic development of solid-shell element formulations for linear and non-linear analyses employing only displacement degrees of freedom, Int. J. Numer. Methods Eng., 42 (1998) 49-69.

[26] D. Lemosse, Eléments finis isoparamétriques tridimensionnels pour l'étude des structures minces, $\mathrm{PhD}$ thesis, INSA-Rouen, France (2000).

[27] K. Y. Sze and L. Q. Yao, A hybrid stress ANS solid-shell element and its generalization for smart structure modeling, Part I-solid-shell element formulation, Int. J. Numer. Methods Eng., 48 (2000) 545-564.

[28] R. Hauptmann, S. Doll, M. Harnau and K. Schweizerhof, Solid-shell elements with linear and quadratic shape functions at large deformations with nearly incompressible mate- 
rials, Comput. Struct., 79 (2001) 1671-1685.

[29] K. Y. Sze and W. K. Chan, A six-node pentagonal assumed natural strain solid-shell element, Finite Elements Anal. Des., 37 (2001) 639-655.

[30] F. Abed-Meraim, A. Combescure, SHB8PS - a new adaptive, assumed-strain continuum mechanics shell element for impact analysis, Comput. Struct., 80 (2002) 791-803.

[31] F. Abed-Meraim, A. Combescure, An improved assumed strain solid-shell element formulation with physical stabilization for geometric non-linear applications and elasticplastic stability analysis, Int. J. Numer. Methods Eng., 80 (2009) 1640-1686.

[32] A. Legay and A. Combescure, Elastoplastic stability analysis of shells using the physically stabilized finite element SHB8PS, Int. J. Numer. Methods Eng., 57 (2003) 1299-1322.

[33] L. Vu-Quoc and X. G. Tan, Optimal solid shells for nonlinear analyses of multilayer composites, I. Statics, Comput. Methods Appl. Mech. Eng., 192 (2003) 975-1016.

[34] K. Y. Sze, X. H. Liu and S. H. Lo, Hybrid-stress six-node prismatic elements, Int. J. Numer. Methods Eng., 61 (2004) 1451-1470.

[35] Y. I. Chen and G. Y. Wu, A mixed 8-node hexahedral element based on the $\mathrm{Hu}-$ Washizu principle and the field extrapolation technique, Struct. Eng. Mech., 17 (2004) 113-140.

[36] K. D. Kim, G. Z. Liu and S. C. Han, A resultant 8-node solid-shell element for geometrically nonlinear analysis, Comput. Mech., 35 (2005) 315-331.

[37] R. J. Alves de Sousa, R. P. R. Cardoso, R. A. Fontes Valente, J. W. Yoon, J. J. Gracio and R. M. Natal Jorge, A new one-point quadrature enhanced assumed strain (EAS) solid-shell element with multiple integration points along thickness: Part I - Geometrically linear applications, Int. J. Numer. Methods Eng., 62 (2005) 952-977.

[38] R. J. Alves de Sousa, R. P. R. Cardoso, R. A. Fontes Valente, J. W. Yoon, J. J. Gracio and R. M. Natal Jorge, A new one-point quadrature enhanced assumed strain (EAS) solid-shell element with multiple integration points along thickness: Part II - Nonlinear applications, Int. J. Numer. Methods Eng., 67 (2006) 160-188.

[39] R. J. Alves de Sousa, J. W. Yoon, R. P. R. Cardoso, R. A. Fontes Valente and J. J. Gracio, On the use of a reduced enhanced solid-shell (RESS) element for sheet forming simulations, Int. J. Plasticity, 23 (2007) 490-515.

[40] S. Reese, A large deformation solid-shell concept based on reduced integration with hourglass stabilization, Int. J. Numer. Methods Eng., 69 (2007) 1671-1716.

[41] J. C. Simo and M. S. Rifai, A class of mixed assumed strain methods and the method of incompatible modes, Int. J. Numer. Methods Eng., 29 (1990) 1595-1638.

[42] J. C. Simo and F. Armero, Geometrically non-linear enhanced strain mixed methods and the method of incompatible modes, Int. J. Numer. Methods Eng., 33 (1992) 1413-1449.

[43] J. C. Simo, F. Armero and R. L. Taylor, Improved versions of assumed enhanced strain tri- linear elements for 3D finite deformation problems, Comput. Methods Appl. Mech. Eng., 110 (1993) 359-386.

[44] E. N. Dvorkin and K. J. Bathe, Continuum mechanics based four-node shell element for general non-linear analysis, Eng. Comput., 1 (1984) 77-88.

[45] T. Belytschko and L. P. Bindeman, Assumed strain stabilization of the eight node hexahedral element, Comput. Methods Appl. Mech. Eng., 105 (1993) 225-260.

[46] Y. Y. Zhu and S. Cescotto, Unified and mixed formulation of the 8-node hexahedral elements by assumed strain method, Comput. Methods Appl. Mech. Eng., 129 (1996) 177-209.

[47] P. Wriggers and S. Reese, A note on enhanced strain methods for large deformations, Comput. Methods Appl. Mech. Eng., 135 (1996) 201-209.

[48] S. Klinkel and W. Wagner, A geometrical non-linear brick element based on the EAS-method, Int. J. Numer. Methods Eng., 40 (1997) 4529-4545.

[49] S. Klinkel, F. Gruttmann and W. Wagner, A continuum based three-dimensional shell element for laminated structures, Comput. Struct., 71 (1999) 43-62.

[50] W. A. Wall, M. Bischoff and E. Ramm, A deformation dependent stabilization technique, exemplified by EAS elements at large strains, Comput. Methods Appl. Mech. Eng., 188 (2000) 859-871.

[51] S. Reese, P. Wriggers and B. D. Reddy, A new locking-free brick element technique for large deformation problems in elasticity, Comput. Struct., 75 (2000) 291-304.

[52] M. A. Puso, A highly efficient enhanced assumed strain physically stabilized hexahedral element, Int. J. Numer. Methods Eng., 49 (2000) 1029-1064.

[53] O. C. Zienkiewicz, R. L. Taylor and J. M. Too, Reduced integration technique in general analysis of plates and shells, Int. J. Numer. Methods Eng., 3 (1971) 275-290.

[54] S. Pawsey and R. Clough, Improved numerical integration of thick shell finite elements, Int. J. Numer. Methods Eng., 3 (1971) 575-586.

[55] T. J. R. Hughes, R. L. Taylor and W. Kanoknukulchai, A simple and efficient finite element for plate bending, Int. J. Numer. Methods Eng., 11 (1977) 1529-1543.

[56] D. P. Flanagan and T. Belytschko, A uniform strain hexahedron and quadrilateral with orthogonal hourglass control, Int. J. Numer. Methods Eng., 17 (1981) 679-706.

[57] J. O. Hallquist, Theoretical manual for DYNA3D, Report UC1D-19401, Lawrence Livermore National Laboratory, Livermore, CA, USA (1983).

[58] J. Fish and T. Belytschko, Elements with embedded localization zones for large deformation problems, Comput. Struct., 30 (1988) 247-256.

[59] J. C. Simo and T. J. R. Hughes, On the variational foundations of assumed strain methods, ASME J. Appl. Mech., 53 (1986) 51-54.

[60] R. H. MacNeal and R. L. Harder, A proposed standard set of problems to test finite element accuracy, Finite Elements Anal. Des., 1 (1985) 3-20.

[61] J. L. Batoz and G. Dhatt, Modelling of structures by finite 
elements, Paris: Hermès Science (1992).

[62] T. Belytschko, B. L. Wong and H. Stolarski, Assumed strain stabilization procedure for the 9-node Lagrange shell element, Int. J. Numer. Methods Eng., 28 (1989) 385-414.

[63] A. Legay, Une méthode de calcul efficace pour l'étude paramétrique du flambage non-linéaire de structures tridimensionnelles : application à la fiabilité. $\mathrm{PhD}$ thesis, LMT-ENS-Cachan, France (2002).

[64] J. M. Bourinet, N. Gayton and M. Lemaire, A. Combescure, Reliability analysis of stability of shells based on combined finite element and response surface methods, In: M. Papadrakis, A. Samartin, E. Onate, editors. Computational Methods for Shell and Spatial Structures, IASS-IACM, Athens, Greece (2000).

[65] I. Leahu-Aluas and F. Abed-Meraim, A proposed set of popular limit-point buckling benchmark problems, Struct. Eng. Mech., 38 (2011) 767-802.

[66] M. Killpack and F. Abed-Meraim, Limit-point buckling analyses using solid, shell and solid-shell elements, J. Mech. Sci. Tech., 25 (2011) 1105-1117.

\section{Appendix}

\section{A.1 Calculation of the constant gradients: vectors $\mathbf{b}_{i}$}

In this appendix, the explicit expressions for the derivatives of the shape functions evaluated at the origin of the reference coordinate system, as given by Eq. (9), will be derived. This particular form of the vectors $\mathbf{b}_{i}$, referred to as Hallquist's form, reads:

$$
\mathbf{b}_{i}^{T}=\left.\mathbf{N}_{, i}^{T}\right|_{\xi=\eta=\zeta=0}=\left(\frac{\partial N_{1}}{\partial x_{i}} \frac{\partial N_{2}}{\partial x_{i}} \frac{\partial N_{3}}{\partial x_{i}} \frac{\partial N_{4}}{\partial x_{i}} \frac{\partial N_{5}}{\partial x_{i}} \frac{\partial N_{6}}{\partial x_{i}}\right)_{\mid \xi=\eta=\zeta=0} .
$$

(A1)

Each of the six components of the above vector can be expressed as:

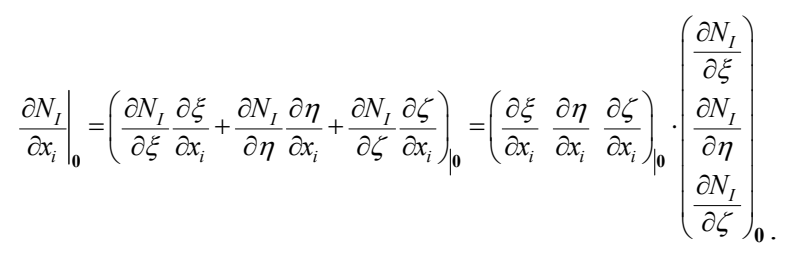

From Eq. (4), the derivatives $\frac{\partial N_{I}}{\partial \xi}$, where $\xi^{T}=(\xi \eta \zeta)$, are straightforward:

$$
\left(\frac{\partial \mathbf{N}}{\partial \xi}\right)^{T}=\frac{1}{2}\left(\begin{array}{cccccc}
-(1-\zeta) & (1-\zeta) & 0 & -(1+\zeta) & (1+\zeta) & 0 \\
-(1-\zeta) & 0 & (1-\zeta) & -(1+\zeta) & 0 & (1+\zeta) \\
-(1-\xi-\eta) & -\xi & -\eta & (1-\xi-\eta) & \xi & \eta
\end{array}\right)
$$

which gives:

$$
\left(\frac{\partial \mathbf{N}}{\partial \xi}\right)_{\mid \mathbf{0}}^{T}=\frac{1}{2}\left(\begin{array}{cccccc}
-1 & 1 & 0 & -1 & 1 & 0 \\
-1 & 0 & 1 & -1 & 0 & 1 \\
-1 & 0 & 0 & 1 & 0 & 0
\end{array}\right) .
$$

For the remaining terms, $\frac{\partial \xi_{j}}{\partial x_{i}}$, further derivations are needed. This requires the calculation of matrix $\mathbf{J}$ such that:

$$
\mathbf{J}=\left(\frac{\partial \xi}{\partial \mathbf{x}}\right)^{T}=\left(\begin{array}{lll}
\frac{\partial \xi}{\partial x} & \frac{\partial \eta}{\partial x} & \frac{\partial \zeta}{\partial x} \\
\frac{\partial \xi}{\partial y} & \frac{\partial \eta}{\partial y} & \frac{\partial \zeta}{\partial y} \\
\frac{\partial \xi}{\partial z} & \frac{\partial \eta}{\partial z} & \frac{\partial \zeta}{\partial z}
\end{array}\right) .
$$

Once this matrix $\mathbf{J}$ known, the Hallquist form of vectors $\mathbf{b}_{i}$ is simply obtained by:

$$
\left(\begin{array}{c}
\mathbf{b}_{1}^{T} \\
\mathbf{b}_{2}^{T} \\
\mathbf{b}_{3}^{T}
\end{array}\right)=\mathbf{J}_{\mid \mathbf{0}} \cdot\left(\frac{\partial \mathbf{N}}{\partial \xi}\right)_{\mid \mathbf{0}}^{T} .
$$

Using the Jacobian matrix and its inverse, matrix $\mathbf{J}$ can be rewritten as:

$$
\begin{gathered}
\mathbf{J}=\left(\frac{\partial \xi}{\partial \mathbf{x}}\right)^{T}=\left(\mathbf{F}^{-1}\right)^{T}=\left(\mathbf{F}^{T}\right)^{-1}, \\
\text { where } \mathbf{F}=\frac{\partial \mathbf{x}}{\partial \xi}=\left(\begin{array}{ccc}
\frac{\partial x}{\partial \xi} & \frac{\partial x}{\partial \eta} & \frac{\partial x}{\partial \zeta} \\
\frac{\partial y}{\partial \xi} & \frac{\partial y}{\partial \eta} & \frac{\partial y}{\partial \zeta} \\
\frac{\partial z}{\partial \xi} & \frac{\partial z}{\partial \eta} & \frac{\partial z}{\partial \zeta}
\end{array}\right) .
\end{gathered}
$$

Making use of Eq. (1), the element coordinate interpolation can be expanded as:

$$
x_{i}=\frac{1}{2}\left(\mathbf{s}_{1}^{T}+\xi \Lambda_{1}^{T}+\eta \Lambda_{2}^{T}+\zeta \Lambda_{3}^{T}+h_{1} \boldsymbol{\beta}_{1}^{T}+h_{2} \boldsymbol{\beta}_{2}^{T}\right) \cdot \mathbf{x}_{i} \quad ; \quad i=1,2,3
$$

where:

$$
\left\{\begin{array}{l}
\mathbf{s}_{1}^{T}=(1,0,0,1,0,0) \\
\Lambda_{1}^{T}=(-1,1,0,-1,1,0) \\
\Lambda_{2}^{T}=(-1,0,1,-1,0,1) \\
\Lambda_{3}^{T}=(-1,0,0,1,0,0) \\
\boldsymbol{\beta}_{1}^{T}=(1,0,-1,-1,0,1) \\
\boldsymbol{\beta}_{2}^{T}=(1,-1,0,-1,1,0) .
\end{array}\right.
$$


The components of the Jacobian matrix are then obtained by differentiation:

$$
F_{i j}=\frac{\partial x_{i}}{\partial \xi_{j}}=\frac{1}{2}\left(\Lambda_{j}^{T}+h_{\alpha, \xi_{j}} \boldsymbol{\beta}_{\alpha}^{T}\right) \cdot \mathbf{x}_{i} \quad ; \quad i, j=1, \ldots, 3 \quad \alpha=1,2
$$

which gives:

$$
\mathbf{F}_{\mid \mathbf{0}}=\frac{1}{2}\left(\begin{array}{ccc}
\boldsymbol{\Lambda}_{1}^{T} \cdot \mathbf{x}_{1} & \boldsymbol{\Lambda}_{2}^{T} \cdot \mathbf{x}_{1} & \boldsymbol{\Lambda}_{3}^{T} \cdot \mathbf{x}_{1} \\
\boldsymbol{\Lambda}_{1}^{T} \cdot \mathbf{x}_{2} & \boldsymbol{\Lambda}_{2}^{T} \cdot \mathbf{x}_{2} & \boldsymbol{\Lambda}_{3}^{T} \cdot \mathbf{x}_{2} \\
\boldsymbol{\Lambda}_{1}^{T} \cdot \mathbf{x}_{3} & \boldsymbol{\Lambda}_{2}^{T} \cdot \mathbf{x}_{3} & \boldsymbol{\Lambda}_{3}^{T} \cdot \mathbf{x}_{3}
\end{array}\right)
$$

and hence:

$$
\mathbf{J}_{\mid \mathbf{0}}=\left(\mathbf{F}^{T}\right)_{\mid \mathbf{0}}^{-1}=2\left(\begin{array}{ccc}
\boldsymbol{\Lambda}_{1}^{T} \cdot \mathbf{x}_{1} & \boldsymbol{\Lambda}_{1}^{T} \cdot \mathbf{x}_{2} & \boldsymbol{\Lambda}_{1}^{T} \cdot \mathbf{x}_{3} \\
\boldsymbol{\Lambda}_{2}^{T} \cdot \mathbf{x}_{1} & \boldsymbol{\Lambda}_{2}^{T} \cdot \mathbf{x}_{2} & \boldsymbol{\Lambda}_{2}^{T} \cdot \mathbf{x}_{3} \\
\boldsymbol{\Lambda}_{3}^{T} \cdot \mathbf{x}_{1} & \boldsymbol{\Lambda}_{3}^{T} \cdot \mathbf{x}_{2} & \boldsymbol{\Lambda}_{3}^{T} \cdot \mathbf{x}_{3}
\end{array}\right)^{-1}
$$

The Hallquist form of vectors $\mathbf{b}_{i}$ is finally given by:

$$
\begin{aligned}
& \left(\begin{array}{l}
\mathbf{b}_{1}^{T} \\
\mathbf{b}_{2}^{T} \\
\mathbf{b}_{3}^{T}
\end{array}\right)=\mathbf{J}_{\mid 0} \cdot\left(\frac{\partial \mathbf{N}}{\partial \xi}\right)_{\mid 0}^{T}= \\
& \left(\begin{array}{lll}
\boldsymbol{\Lambda}_{1}^{T} \cdot \mathbf{x}_{1} & \boldsymbol{\Lambda}_{1}^{T} \cdot \mathbf{x}_{2} & \boldsymbol{\Lambda}_{1}^{T} \cdot \mathbf{x}_{3} \\
\boldsymbol{\Lambda}_{2}^{T} \cdot \mathbf{x}_{1} & \boldsymbol{\Lambda}_{2}^{T} \cdot \mathbf{x}_{2} & \boldsymbol{\Lambda}_{2}^{T} \cdot \mathbf{x}_{3} \\
\boldsymbol{\Lambda}_{3}^{T} \cdot \mathbf{x}_{1} & \boldsymbol{\Lambda}_{3}^{T} \cdot \mathbf{x}_{2} & \boldsymbol{\Lambda}_{3}^{T} \cdot \mathbf{x}_{3}
\end{array}\right)^{-1} \cdot\left(\begin{array}{cccccc}
-1 & 1 & 0 & -1 & 1 & 0 \\
-1 & 0 & 1 & -1 & 0 & 1 \\
-1 & 0 & 0 & 1 & 0 & 0
\end{array}\right) .
\end{aligned}
$$

Using this expression (A13) of vectors $\mathbf{b}_{i}$, the orthogonality conditions given in Eq. (10) become algebraically straightforward. The last consistency condition follows simply from $\mathbf{b}_{i}^{T} \cdot \mathbf{x}_{j}=\mathbf{N}_{, i \mid \mathbf{0}}^{T} \cdot \mathbf{x}_{j}=x_{j, i \mid \mathbf{0}}=\delta_{i j}$.

\section{A.2 Analysis of hourglass modes}

As mentioned in Section 2.4, the hourglass patterns in subintegrated finite elements correspond to zero-energy modes, i.e., eigenvectors associated with zero-eigenvalues, aside from rigid body modes. In order to properly identify the kernel of the stiffness matrix, the orthogonality conditions stated in Eq. (10) will be used together with some algebraic derivations. Since the discrete stiffness matrix is given by Eq. (29), a zeroenergy mode is a vector $\mathbf{h}^{g}$ that satisfies:

$$
\mathbf{B}\left(\zeta_{I}\right) \cdot \mathbf{h}^{g}=\mathbf{0} \quad ; \quad I=1, \ldots, n_{\mathrm{int}}
$$

It can be easily shown that a basis for the vector space of the discretized displacements is given by the following vectors:

$$
\begin{aligned}
& \mathbf{e}_{1}=\left(\begin{array}{l}
\mathbf{s} \\
\mathbf{0} \\
\mathbf{0}
\end{array}\right), \mathbf{e}_{2}=\left(\begin{array}{l}
\mathbf{0} \\
\mathbf{s} \\
\mathbf{0}
\end{array}\right), \mathbf{e}_{3}=\left(\begin{array}{l}
\mathbf{0} \\
\mathbf{0} \\
\mathbf{s}
\end{array}\right), \mathbf{e}_{4}=\left(\begin{array}{l}
\mathbf{x} \\
\mathbf{0} \\
\mathbf{0}
\end{array}\right), \mathbf{e}_{5}=\left(\begin{array}{l}
\mathbf{0} \\
\mathbf{x} \\
\mathbf{0}
\end{array}\right), \\
& \mathbf{e}_{6}=\left(\begin{array}{l}
\mathbf{0} \\
\mathbf{0} \\
\mathbf{x}
\end{array}\right), \mathbf{e}_{7}=\left(\begin{array}{l}
\mathbf{y} \\
\mathbf{0} \\
\mathbf{0}
\end{array}\right), \mathbf{e}_{8}=\left(\begin{array}{l}
\mathbf{0} \\
\mathbf{y} \\
\mathbf{0}
\end{array}\right), \mathbf{e}_{9}=\left(\begin{array}{l}
\mathbf{0} \\
\mathbf{0} \\
\mathbf{y}
\end{array}\right), \mathbf{e}_{10}=\left(\begin{array}{l}
\mathbf{z} \\
\mathbf{0} \\
\mathbf{0}
\end{array}\right), \\
& \mathbf{e}_{11}=\left(\begin{array}{l}
\mathbf{0} \\
\mathbf{z} \\
\mathbf{0}
\end{array}\right), \mathbf{e}_{12}=\left(\begin{array}{c}
\mathbf{0} \\
\mathbf{0} \\
\mathbf{z}
\end{array}\right), \mathbf{e}_{13}=\left(\begin{array}{c}
\mathbf{h}_{1} \\
\mathbf{0} \\
\mathbf{0}
\end{array}\right), \mathbf{e}_{14}=\left(\begin{array}{c}
\mathbf{0} \\
\mathbf{h}_{1} \\
\mathbf{0}
\end{array}\right), \\
& \mathbf{e}_{15}=\left(\begin{array}{c}
\mathbf{0} \\
\mathbf{0} \\
\mathbf{h}_{1}
\end{array}\right), \mathbf{e}_{16}=\left(\begin{array}{c}
\mathbf{h}_{2} \\
\mathbf{0} \\
\mathbf{0}
\end{array}\right), \mathbf{e}_{17}=\left(\begin{array}{c}
\mathbf{0} \\
\mathbf{h}_{2} \\
\mathbf{0}
\end{array}\right), \mathbf{e}_{18}=\left(\begin{array}{c}
\mathbf{0} \\
\mathbf{0} \\
\mathbf{h}_{2}
\end{array}\right) .
\end{aligned}
$$

To demonstrate this, let us assume that there exists a combination of coefficients $a_{i}$ such that:

$$
\sum_{i=1}^{18} a_{i} \mathbf{e}_{i}=\mathbf{0}
$$

Multiplying Eq. (A16) by $\left(\begin{array}{lll}\mathbf{b}_{i}^{T} & \mathbf{0} & \mathbf{0}\end{array}\right),\left(\begin{array}{lll}\mathbf{0} & \mathbf{b}_{i}^{T} & \mathbf{0}\end{array}\right)$, and ( $\left.\begin{array}{lll}\mathbf{0} & \mathbf{0} & \mathbf{b}_{i}^{T}\end{array}\right)$, for $i=1,2,3$, successively, and making use of Eq. (10) yields:

$$
\left\{\begin{array}{l}
\left(\begin{array}{lll}
\mathbf{b}_{i}^{T} & \mathbf{0} & \mathbf{0}
\end{array}\right) \cdot\left(\sum_{i=1}^{18} a_{i} \mathbf{e}_{i}\right)=a_{3 i+1}=0 \\
\left(\begin{array}{lll}
\mathbf{0} & \mathbf{b}_{i}^{T} & \mathbf{0}
\end{array}\right) \cdot\left(\sum_{i=1}^{18} a_{i} \mathbf{e}_{i}\right)=a_{3 i+2}=0 \quad, \quad i=1,2,3 \\
\left(\begin{array}{lll}
\mathbf{0} & \mathbf{0} & \mathbf{b}_{i}^{T}
\end{array}\right) \cdot\left(\sum_{i=1}^{18} a_{i} \mathbf{e}_{i}\right)=a_{3 i+3}=0 .
\end{array}\right.
$$

Repeating this operation with $\left(\begin{array}{lll}\mathbf{h}_{\alpha}^{T} & \mathbf{0} & \mathbf{0}\end{array}\right),\left(\begin{array}{lll}\mathbf{0} & \mathbf{h}_{\alpha}^{T} & \mathbf{0}\end{array}\right)$, and $\left(\begin{array}{lll}\mathbf{0} & \mathbf{0} & \mathbf{h}_{\alpha}^{T}\end{array}\right)$, for $\alpha=1,2$, successively, yields:

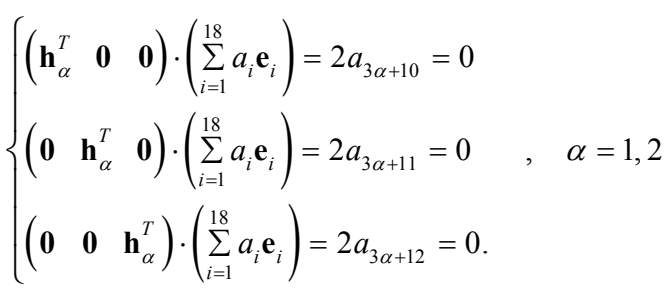

Combining Eqs. (A15-A18), it becomes obvious that:

$$
a_{1}=a_{2}=a_{3}=0
$$

This shows that vectors $\left(\mathbf{e}_{i}, i=1, \ldots, 18\right)$ are linearly independent, and hence, they form a basis for the vector space of discretized displacements.

Let us now assume that vector $\mathbf{h}^{g}$ belongs to the kernel of 
the stiffness matrix. This vector can be expanded in terms of the above base vectors as:

$$
\mathbf{h}^{g}=\sum_{i=1}^{18} c_{i} \mathbf{e}_{i}
$$

Using Eq. (A14) together with Eq. (15) and orthogonality condition (10) yields:

$$
\begin{gathered}
\left(\begin{array}{c}
c_{4}+h_{1, x}\left(\xi_{I}\right) c_{13}+h_{2, x}\left(\xi_{I}\right) c_{16} \\
c_{8}+h_{1, y}\left(\xi_{I}\right) c_{14}+h_{2, y}\left(\xi_{I}\right) c_{17} \\
c_{12}+h_{1, z}\left(\xi_{I}\right) c_{15}+h_{2, z}\left(\xi_{I}\right) c_{18} \\
c_{5}+c_{7}+h_{1, y}\left(\xi_{I}\right) c_{13}+h_{1, x}\left(\xi_{I}\right) c_{14}+h_{2, y}\left(\xi_{I}\right) c_{16}+h_{2, x}\left(\xi_{I}\right) c_{17} \\
c_{9}+c_{11}+h_{1, z}\left(\xi_{I}\right) c_{14}+h_{1, y}\left(\xi_{I}\right) c_{15}+h_{2, z}\left(\xi_{I}\right) c_{17}+h_{2, y}\left(\xi_{I}\right) c_{18} \\
c_{6}+c_{10}+h_{1, z}\left(\xi_{I}\right) c_{13}+h_{1, x}\left(\xi_{I}\right) c_{15}+h_{2, z}\left(\xi_{I}\right) c_{16}+h_{2, x}\left(\xi_{I}\right) c_{18}
\end{array}\right)=\mathbf{0} \\
I=1, \ldots, h_{\text {int }} .
\end{gathered}
$$

Evaluating the above equation at the $n_{\text {int }}$ different Gauss points implies that:

$$
\left\{\begin{array}{l}
c_{4}=c_{13}=c_{16}=0 \\
c_{8}=c_{14}=c_{17}=0 \\
c_{12}=c_{15}=c_{18}=0 \\
c_{5}+c_{7}=0 \\
c_{9}+c_{11}=0 \\
c_{6}+c_{10}=0
\end{array}\right.
$$

and hence:

$$
\mathbf{h}^{g}=c_{1}\left(\begin{array}{l}
\mathbf{s} \\
\mathbf{0} \\
\mathbf{0}
\end{array}\right)+c_{2}\left(\begin{array}{l}
\mathbf{0} \\
\mathbf{s} \\
\mathbf{0}
\end{array}\right)+c_{3}\left(\begin{array}{l}
\mathbf{0} \\
\mathbf{0} \\
\mathbf{s}
\end{array}\right)+c_{5}\left(\begin{array}{r}
-\mathbf{y} \\
\mathbf{x} \\
\mathbf{0}
\end{array}\right)+c_{6}\left(\begin{array}{r}
-\mathbf{z} \\
\mathbf{0} \\
\mathbf{x}
\end{array}\right)+c_{9}\left(\begin{array}{r}
\mathbf{0} \\
-\mathbf{z} \\
\mathbf{y}
\end{array}\right) .
$$

This reveals that the kernel of the stiffness matrix for the SHB6 element only consists of the usual six rigid body modes. It should be noted that this formulation of the SHB6 element is valid for any set of $n_{\text {int }}$ integration points located along the same line $\xi_{I}=\eta_{I}=\frac{1}{3}, \zeta_{I}, \quad I=1, \ldots, n_{\text {int }}$, and comprising at least two Gauss points $\left(n_{\text {int }} \geq 2\right)$. In this case, Eq. (A23) clearly shows that only rigid body modes belong to the kernel of the stiffness matrix, and thus no rank deficiency is observed.

\section{A.3 Geometric stiffness matrix for buckling analysis}

In this appendix, the geometric stiffness matrix is derived for the SHB6 element. This geometric stiffness matrix $\mathbf{K}_{\sigma}$ is to be added to the regular tangent stiffness matrix $\mathbf{K}$ in a usual structural stability analysis; see Eq. (42), for instance.
Recall that the geometric stiffness matrix originates from the linearization of the virtual work principle and is due to the nonlinear (quadratic) part of the strain tensor. In its continuum form, it reads:

$$
K_{\sigma}(\delta \mathbf{u}, \Delta \mathbf{u})=\int_{\Omega_{e}} \sigma: \nabla \delta \mathbf{u}^{T} \cdot \nabla \Delta \mathbf{u} d \Omega=\int_{\Omega_{e}} \sigma: \mathbf{e}^{Q}(\delta \mathbf{u}, \Delta \mathbf{u}) d \Omega .
$$

Using the vector form of the stress tensor and the quadratic part of the strain tensor, respectively, Eq. (A24) can be rewritten as:

$$
K_{\boldsymbol{\sigma}}(\delta \mathbf{u}, \Delta \mathbf{u})=\int_{\Omega_{e}} \boldsymbol{\sigma}^{T} \cdot \mathbf{e}^{Q}(\delta \mathbf{u}, \Delta \mathbf{u}) d \Omega
$$

where:

$$
\boldsymbol{\sigma}=\left[\begin{array}{c}
\sigma_{x x} \\
\sigma_{y y} \\
\sigma_{z z} \\
\sigma_{x y} \\
\sigma_{y z} \\
\sigma_{x z}
\end{array}\right] \quad, \quad \mathbf{e}^{Q}=\left[\begin{array}{c}
e_{x x}^{Q} \\
e_{y y}^{Q} \\
e_{z z}^{Q} \\
e_{x y}^{Q}+e_{y x}^{Q} \\
e_{y z}^{Q}+e_{z y}^{Q} \\
e_{x z}^{Q}+e_{z x}^{Q}
\end{array}\right]
$$

and the components of the quadratic part of the strain tensor are given by:

$$
e_{i j}^{Q}(\delta \mathbf{u}, \Delta \mathbf{u})=\sum_{k=1}^{3} \delta u_{k, i} \Delta u_{k, j}=\delta u_{k, i} \Delta u_{k, j} .
$$

Using the discrete form of the displacement gradient, as given in Eq. (13), one obtains:

$$
\left\{\begin{array}{l}
\delta u_{k, i}=\left(\mathbf{b}_{i}^{T}+h_{\alpha, i} \gamma_{\alpha}^{T}\right) \cdot \delta \mathbf{d}_{k}=\mathbf{B}_{i}^{T} \cdot \delta \mathbf{d}_{k} \\
\Delta u_{k, j}=\left(\mathbf{b}_{j}^{T}+h_{\alpha, j} \gamma_{\alpha}^{T}\right) \cdot \Delta \mathbf{d}_{k}=\mathbf{B}_{j}^{T} \cdot \Delta \mathbf{d}_{k}
\end{array}\right.
$$

The components of the quadratic part of the strain tensor can then be discretized as:

$$
e_{i j}^{Q}(\delta \mathbf{u}, \Delta \mathbf{u})=\sum_{k=1}^{3}\left(\delta \mathbf{d}_{k}^{T} \cdot \mathbf{B}_{i}\right)\left(\mathbf{B}_{j}^{T} \cdot \Delta \mathbf{d}_{k}\right)=\delta \mathbf{d}^{T} \cdot \mathbf{B}_{i j}^{Q} \cdot \Delta \mathbf{d}
$$

where:

$$
\mathbf{B}_{i j}^{Q}=\left[\begin{array}{ccc}
\mathbf{B}_{i} \mathbf{B}_{j}^{T} & \mathbf{0} & \mathbf{0} \\
\mathbf{0} & \mathbf{B}_{i} \mathbf{B}_{j}^{T} & \mathbf{0} \\
\mathbf{0} & \mathbf{0} & \mathbf{B}_{i} \mathbf{B}_{j}^{T}
\end{array}\right], \delta \mathbf{d}=\left[\begin{array}{c}
\delta \mathbf{d}_{1} \\
\delta \mathbf{d}_{2} \\
\delta \mathbf{d}_{3}
\end{array}\right], \Delta \mathbf{d}=\left[\begin{array}{c}
\Delta \mathbf{d}_{1} \\
\Delta \mathbf{d}_{2} \\
\Delta \mathbf{d}_{3}
\end{array}\right] .
$$


With these quadratic discrete gradient operators $\mathbf{B}_{i j}^{Q}$, the contribution $\mathbf{k}_{\sigma}\left(\zeta_{I}\right)$ at Gauss point $\zeta_{I}$ to the overall geometric stiffness matrix is given by:

$$
\begin{aligned}
& \mathbf{k}_{\sigma}\left(\zeta_{I}\right)=\sigma_{x x}\left(\zeta_{I}\right) \mathbf{B}_{x x}^{Q}\left(\zeta_{I}\right)+\sigma_{y y}\left(\zeta_{I}\right) \mathbf{B}_{y y}^{Q}\left(\zeta_{I}\right) \\
&+\sigma_{z z}\left(\zeta_{I}\right) \mathbf{B}_{z z}^{Q}\left(\zeta_{I}\right)+\sigma_{x y}\left(\zeta_{I}\right)\left(\mathbf{B}_{x y}^{Q}\left(\zeta_{I}\right)+\mathbf{B}_{y x}^{Q}\left(\zeta_{I}\right)\right) \\
&+\sigma_{y z}\left(\zeta_{I}\right)\left(\mathbf{B}_{y z}^{Q}\left(\zeta_{I}\right)+\mathbf{B}_{z y}^{Q}\left(\zeta_{I}\right)\right) \\
&+\sigma_{x z}\left(\zeta_{I}\right)\left(\mathbf{B}_{x z}^{Q}\left(\zeta_{I}\right)+\mathbf{B}_{z x}^{Q}\left(\zeta_{I}\right)\right) .
\end{aligned}
$$

The geometric stiffness matrix is finally obtained by Gauss integration as:

$$
\mathbf{K}_{\sigma}=\sum_{I=1}^{n_{\text {int }}} \omega\left(\zeta_{I}\right) J\left(\zeta_{I}\right) \mathbf{k}_{\sigma}\left(\zeta_{I}\right)
$$

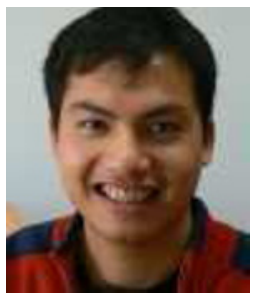

Vuong-Dieu Trinh graduated as a Civil Engineer from the National Civil Engineering School, Ha Noi, Vietnam in 2001. He received his M.S. in Structural Mechanics from École Nationale des Ponts et Chaussées, Paris in 2003 and his Ph.D in Structural and Applied Mechanics from Arts et Métiers ParisTech, Metz Campus in 2008. He is currently working as a Civil Engineer at Peikko Group Corporation, an international company.

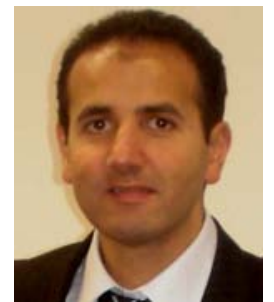

Farid Abed-Meraim received his $\mathrm{Ph} . \mathrm{D}$ in Theoretical and Applied Mechanics from École Polytechnique, Paris in 1999. He joined Arts et Métiers ParisTech, Metz Campus in 2000, where he is currently an Associate Professor at the LEM3 laboratory. His main research interests include structural stability (bifurcation) analysis of dissipative systems (elasto-plastic, visco-elastic and visco-plastic), material instability modeling in relation to the prediction of formability of metal sheets, as well as finite element technology (solid-shell formulations).

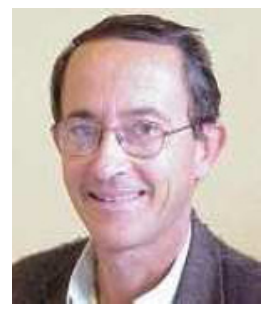

Alain Combescure graduated from École Polytechnique, Paris in 1973. After 20 years at the French Atomic Commission as a research engineer and team leader, working on the simulation of buckling under complex loads, he joined the LMT laboratory at ENS Cachan, where he has been working for six years as a Professor in the field of transient non-linear Mechanics. He was then appointed Professor at INSA Lyon and head of the LaMCoS laboratory. His main research interests concern fracture mechanics, phase transformation in metals, time space subdomain coupling, and X-FEM methods for transient crack propagation. 